\title{
On wormholes in the moduli space of surfaces
}

\author{
Giancarlo Urzúa and Nicolás Vilches
}

\begin{abstract}
We study a certain wormholing phenomenon that takes place in the Kollár-ShepherdBarron-Alexeev (KSBA) compactification of the moduli space of surfaces of general type. It occurs because of the appearance of particular extremal P-resolutions in surfaces on the KSBA boundary. We state a general wormhole conjecture, and we prove it for a wide range of cases. At the end, we discuss some topological properties and open questions.
\end{abstract}

\section{Introduction}

Since the breakthrough construction of simply connected Campedelli surfaces by Lee and Park in [LP07], there have been several results on various aspects of 1-parameter $\mathbb{Q}$-Gorenstein degenerations of surfaces; see, for example, [PPS09a, PPS09b, LN13, SU16, HTU17, Urz16b, Urz16a, RTU17, LN18, PPSU18, CU18, EU18]. One of those aspects has been the study of Kollár-Shepherd-Barron-Alexeev (KSBA) surfaces with only Wahl singularities which admit $\mathbb{Q}$-Gorenstein smoothings into surfaces of general type. These smoothings could be seen as punctured disks $\mathbb{D}^{\times}$on the moduli space of surfaces of general type $M_{K^{2}, \chi}$, which are completed in the KSBA compactification $\bar{M}_{K^{2}, \chi}$ with a normal projective surface $X$ with only Wahl singularities and $K_{X}$ ample. (Here of course $K_{X}^{2}=K^{2}$ and $\chi\left(\mathcal{O}_{X}\right)=\chi$.) In this way, we have a $\mathbb{Q}$-Gorenstein smoothing

$$
(X \subset \mathcal{X}) \rightarrow(0 \in \mathbb{D})
$$

where $\mathbb{D}=\overline{\mathbb{D}^{\times}}$. Nowadays there are many examples of such situations in the literature, most of them constructed abstractly, starting with the original work [LP07].

P-resolutions were introduced by Kollár-Shepherd-Barron to classify deformations of quotient singularities [KS88, Section 3]. The smallest ones over cyclic quotient singularities, which are called extremal P-resolutions (see Definition 2.4), play a key role for us in the following sense. Sometimes a surface $X$ as above has an embedded extremal P-resolution, which in addition admits another extremal P-resolution over the same cyclic quotient singularity. One performs the corresponding "extremal P-resolution surgery" on $X$ to obtain another normal projective surface $X^{\prime}$ with only Wahl singularities. Let us assume that $X^{\prime}$ admits a $\mathbb{Q}$-Gorenstein smoothing $\left(X^{\prime} \subset \mathcal{X}^{\prime}\right) \rightarrow(0 \in \mathbb{D})$. (This automatically holds under a cohomological condition on $X$ that

Received 11 February 2021, accepted in final form 27 May 2021.

2020 Mathematics Subject Classification 14J10, 14E30, 14J29, $14 \mathrm{~J} 17$.

Keywords: moduli space of surfaces of general type, KSBA compactification, minimal model program, Wahl singularity, continued fractions.

This journal is (C) Foundation Compositio Mathematica 2022. This article is distributed with Open Access under the terms of the Creative Commons Attribution Non-Commercial License, which permits non-commercial reuse, distribution, and reproduction in any medium, provided that the original work is properly cited. For commercial re-use, please contact the Foundation Compositio Mathematica.

The first-named author was supported by the FONDECYT regular grant 1190066. The second-named author was funded by the ANID scholarship 22190759. 


\section{G. URzÚa AND N. VilcheS}

is used in all Lee-Park type of surfaces.) If $K_{X^{\prime}}$ is ample, then one can easily show that $X$ and $X^{\prime}$ live in the same $\bar{M}_{K^{2}, \chi}$. If $K_{X^{\prime}}$ is only nef, then the canonical models of $X^{\prime}$ and $X$ belong to the same $\bar{M}_{K^{2}, \chi}$ as well. But if $K_{X^{\prime}}$ is not nef, then one needs to run the minimal model program (MMP) on the 3-fold family $\left(X^{\prime} \subset \mathcal{X}^{\prime}\right) \rightarrow(0 \in \mathbb{D})$ to find the KSBA replacement (in case that the smooth fiber is of general type), that is, the canonical model of a new family $\left(X^{\prime \prime} \subset \mathcal{X}^{\prime \prime}\right) \rightarrow(0 \in \mathbb{D})$ such that $K_{X^{\prime \prime}}$ is nef and $K_{X^{\prime \prime}}^{2}>0$. This MMP requires flips and/or divisorial contractions as studied in [HTU17] (see also [Urz16a]). If $\left(X^{\prime} \subset \mathcal{X}^{\prime}\right) \rightarrow(0 \in \mathbb{D})$ has a minimal model (that is, the canonical class becomes nef) and the MMP only requires flips, then the KSBA replacement is again on the same $\bar{M}_{K^{2}, \chi}$.

Conjecture 1.1 (Wormhole conjecture). The MMP requires only flips and gives a minimal model. The KSBA replacement of $\left(X^{\prime} \subset \mathcal{X}^{\prime}\right) \rightarrow(0 \in \mathbb{D})$ lives on the same moduli space as the original $(X \subset \mathcal{X}) \rightarrow(0 \in \mathbb{D})$.

It is not clear if the smooth surfaces in the wormhole are deformation equivalent, that is, belong to the same connected component of $M_{K^{2}, \chi}$. For example, Reid conjectures that there is one component for torsion-free Godeaux surfaces, and we do have wormholes there by means of Lee-Park type of examples, which we do not know how to connect. On the other hand, wormholes applied to elliptic surfaces may change the topology. We show examples of that in Section 6.

In this paper, we prove the wormhole conjecture for a wide range of cases. We point out that a fixed extremal P-resolution in $X$ can produce at most one wormhole because in [HTU17, Section 4], it is proved that a cyclic quotient singularity can admit at most two extremal Presolutions. Additionally, when that happens, both share the same $\delta$ invariant; that is, for these two surfaces, the intersection of the exceptional curve with the canonical class times the indices of the singularities is the same. In this paper, we give simplified and new proofs of both of these facts.

We now state the main theorems, which will imply positive evidence for the wormhole conjecture as a corollary. For the definitions, we refer to Sections 2 and 3.

THEOREM 1.2. Let $Y$ be a nonrational normal projective surface with one cyclic quotient singularity $(Q \in Y)$ which is smooth everywhere else. Assume that $Q$ admits two extremal $P$ resolutions $f_{i}^{+}:\left(C_{i} \subseteq X_{i}\right) \rightarrow(Q \in Y)$, for $i=1,2$, such that the following are satisfied:

- The strict transform in the minimal resolution of $X_{2}$ of the exceptional curve $C_{2}$ for the extremal P-resolution in $X_{2}$ is a $\mathbb{P}^{1}$ with self-intersection -1 .

- The canonical class $K_{X_{1}}$ is nef.

- Both surfaces $X_{i}$ admit $\mathbb{Q}$-Gorenstein smoothings $\left(X_{i} \subseteq \mathcal{X}_{i}\right) \rightarrow(0 \in \mathbb{D})$.

Then, $K_{X_{2}}$ is nef.

THEOREM 1.3. Let $Y$ be a nonrational normal projective surface with one cyclic quotient singularity $(Q \in Y)$ which is smooth everywhere else. Assume that $Q$ admits two extremal $P$ resolutions $f_{i}^{+}:\left(C_{i} \subseteq X_{i}\right) \rightarrow(Q \in Y)$, for $i=1,2$, such that the following are satisfied:

- The strict transform in the minimal resolution of $X_{2}$ of the exceptional curve $C_{2}$ for the extremal $P$-resolution in $X_{2}$ is a $\mathbb{P}^{1}$ with self-intersection -2 .

- The extremal P-resolution in $X_{2}$ has only one singularity.

- The canonical class $K_{X_{1}}$ is nef.

- Both surfaces $X_{i}$ admit $\mathbb{Q}$-Gorenstein smoothings $\left(X_{i} \subseteq \mathcal{X}_{i}\right) \rightarrow(0 \in \mathbb{D})$. 


\section{ON WORMHOLES IN THE MODULI SPACE OF SURFACES}

Then, we only need flips to run the MMP on $\left(X_{2} \subset \mathcal{X}_{2}\right) \rightarrow(0 \in \mathbb{D})$.

We can show via an explicit example that one might indeed need to perform flips in a situation as in Theorem 1.3 (see Section 3). Finally, in Section 6, we briefly show and discuss certain topological aspects of wormholes, ending with some open questions and with what is left to prove the wormhole conjecture. We also present a (combinatorial) potential counterexample.

Corollary 1.4. Let $X$ be a normal projective surface with only Wahl singularities and $K_{X}$ ample. We assume the following:

- The surface $X$ is not rational.

- There is an embedded extremal P-resolution in $X$ such that its contraction $(C \subset X) \rightarrow$ $(Q \in Y)$ admits another extremal P-resolution $\left(C^{\prime} \subset X^{\prime}\right) \rightarrow(Q \in Y)$ as in Theorem 1.2 or 1.3 .

- The cohomology group $H^{2}\left(\widetilde{X}, T_{\widetilde{X}}^{0}(-\log (E+\widetilde{C}))\right)$ vanishes, where $\widetilde{X} \rightarrow X$ is the minimal resolution of $X, E$ is the exceptional divisor, and $\widetilde{C}$ is the strict transform of $C$. Hence, there are $\mathbb{Q}$-Gorenstein smoothings $(X \subseteq \mathcal{X}) \rightarrow(0 \in \mathbb{D})$ and $\left(X^{\prime} \subseteq \mathcal{X}^{\prime}\right) \rightarrow(0 \in \mathbb{D})$.

Then, the KSBA replacement of $\left(X^{\prime} \subset \mathcal{X}^{\prime}\right) \rightarrow(0 \in \mathbb{D})$ lives on the same moduli space as the original $(X \subset \mathcal{X}) \rightarrow(0 \in \mathbb{D})$.

\section{Notation and conventions}

- A $(-m)$-curve is a curve $\Gamma$ isomorphic to $\mathbb{P}^{1}$ with $\Gamma^{2}=-m$.

- On a normal surface, we use the intersection theory for Weil divisors defined by Mumford in [Mum61, Section II(b)].

- If $\phi: X \rightarrow W$ is a birational morphism, then $\operatorname{exc}(\phi)$ is the exceptional divisor.

- A KSBA surface in this paper is a normal projective surface with log-canonical singularities and ample canonical class [KS88].

- Under a birational map, we may keep the notation for a curve and its strict transform.

- For a normal projective surface $Z$, the tangent sheaf is denoted by $T_{Z}^{0}:=\mathcal{H}^{\circ} m_{\mathcal{O}_{Z}}\left(\Omega_{Z}^{1}, \mathcal{O}_{Z}\right)$. If $Z$ is not singular and $D$ is a simple normal crossings divisor on $Z$, then $T_{Z}^{0}$ is the usual rank 2 tangent bundle and $T_{Z}^{0}(-\log (D))$ is the dual of the rank 2 vector bundle of differentials with simple poles along $D$.

\section{A review of continued fractions and extremal P-resolutions}

\subsection{Continued fractions}

Definition 2.1. Given positive integers $a_{1}, a_{2}, \ldots, a_{r}$, we define the Hirzebruch-Jung continued fraction recursively. If $r=1$, then $\left[a_{1}\right]:=a_{1}$. If $r \geqslant 2$ and $\left[a_{2}, \ldots, a_{r}\right] \neq 0$, then we define

$$
\left[a_{1}, \ldots, a_{r}\right]:=a_{1}-\frac{1}{\left[a_{2}, \ldots, a_{r}\right]} .
$$

Note that not every list of positive integers makes sense as a continued fraction; for an example, take $[5,1,2,1]$. On the other hand, if $a_{i} \geqslant 2$ for every $i$, the continued fraction automatically makes sense, and $\left[a_{1}, \ldots, a_{r}\right]>1$ by induction on $r$. If $0<q<n$ are coprime numbers, then there exist unique $a_{i} \geqslant 2$ such that

$$
\left[a_{1}, \ldots, a_{r}\right]=\frac{n}{q}
$$




\section{G. URzúa And N. VilcheS}

To analyze these continued fractions, given $a_{1}, \ldots, a_{r}$, we define sequences by setting $p_{0}=1$, $p_{1}=a_{1}, q_{0}=0, q_{1}=1$, and, for $2 \leqslant i \leqslant r$,

$$
p_{i}=a_{i} p_{i-1}-p_{i-2}, \quad q_{i}=a_{i} q_{i-1}-q_{i-1} .
$$

Inductively, one can show that

$$
\left(\begin{array}{cc}
a_{1} & -1 \\
1 & 0
\end{array}\right) \cdots\left(\begin{array}{cc}
a_{i} & -1 \\
1 & 0
\end{array}\right)=\left(\begin{array}{cc}
p_{i} & -p_{i-1} \\
q_{i} & -q_{i-1}
\end{array}\right)
$$

and also $p_{i} / q_{i}=\left[a_{1}, \ldots, a_{i}\right]$ for every $1 \leqslant i \leqslant r$. We say that $\left\{a_{1}, \ldots, a_{r}\right\}$ is admissible if $p_{i}>0$ for $i<r$. A sequence is admissible if and only if the matrix

$$
\left[\begin{array}{ccccc}
-a_{1} & 1 & & & \\
1 & -a_{2} & 1 & & \\
& 1 & -a_{3} & & \\
& & & \ddots & 1 \\
& & & 1 & -a_{r}
\end{array}\right]
$$

is seminegative definite of rank at least $r-1$ (see, for example, [OW77]). Note that if $a_{i} \geqslant 2$ for all $i$, then the sequence is admissible. If some $a_{i}$ is 1 and $r \geqslant 2$, then

$$
\begin{array}{ll}
\left\{a_{2}-1, a_{3}, \ldots, a_{r}\right\}, & i=1 ; \\
\left\{a_{1}, \ldots, a_{i-2}, a_{i-1}-1, a_{i+1}-1, a_{i+2}, \ldots, a_{r}\right\}, & 1 \leqslant i \leqslant r-1 ; \\
\left\{a_{1}, \ldots, a_{r-2}, a_{r-1}-1\right\}, & i=r
\end{array}
$$

are also admissible. We call this procedure a blow-down. If the original fraction was $n / q$, then the new one is $n / q^{\prime}$ with $q^{\prime} \equiv q(\bmod n)$.

Given an admissible continued fraction $\left[a_{1}, \ldots, a_{r}\right]$, after blowing down every possible entry, we may get two different results, according to the rank of the matrix (2.1). If its rank is $r$, then we get either [1] or a continued fraction $\left[b_{1}, \ldots, b_{s}\right]$ with $b_{j} \geqslant 2$ for every $1 \leqslant j \leqslant s$. Otherwise, we get $[1,1]$ as a final fraction.

We define the zero continued fraction as an admissible continued fraction $\left[a_{1}, \ldots, a_{r}\right]$ whose value is equal to zero. Equivalent, the rank of its matrix $(2.1)$ is $r-1$.

Given a fraction $\left[a_{1}, \ldots, a_{r}\right]=n / q$ with $a_{i} \geqslant 2,0<q<n$, and $q$ and $n$ coprime, the dual fraction is

$$
\frac{n}{n-q}=\left[b_{1}, \ldots, b_{s}\right]
$$

with $b_{j} \geqslant 2$ for all $j$. We have a visual way to compute the dual fractions; cf. [Rie74]. Draw $a_{1}-1$ dots horizontally. Under the rightmost one, draw another horizontal line of $a_{2}-1$ dots, and repeat. For instance, if we apply this to $19 / 11=[2,4,3]$, then we obtain Figure 1.

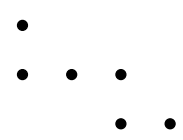

Figure 1. Dot diagram for $[2,4,3]$

Then, we have $b_{1}-1$ dots in the first column, $b_{2}-1$ in the second one, and so on. This shows that $19 / 8=[3,2,3,2]$. 


\section{ON WORMHOLES IN THE MODULI SPACE OF SURFACES}

Suppose $\left[a_{1}, \ldots, a_{r}\right]=n / q$ with $a_{i} \geqslant 2,0<q<n$, and $q$ and $n$ coprime. One can prove that

$$
\left(\begin{array}{cc}
a_{1} & -1 \\
1 & 0
\end{array}\right) \cdots\left(\begin{array}{cc}
a_{i} & -1 \\
1 & 0
\end{array}\right)=\left(\begin{array}{cc}
n & -q^{\prime} \\
q & \left(1-q q^{\prime}\right) / n
\end{array}\right),
$$

where $q^{\prime}$ is the inverse of $q$ modulo $n$, since every matrix on the left has determinant 1 . Thus, if $\left[b_{1}, \ldots, b_{s}\right]=n /(n-q)$ is the unique continued fraction with $b_{j} \geqslant 2$, then

$$
\left[a_{1}, \ldots, a_{r}, 1, b_{1}, \ldots, b_{s}\right]=0 .
$$

2.2 Zero continued fractions. Now we will focus on zero continued fractions, following [Ste91]. Consider a zero continued fraction $\left[a_{1}, \ldots, a_{r}\right]$. Blowing down every possible 1 until the length is 2 , we get $[1,1]$. Reversing the process, every zero continued fraction can be obtained from $[1,1]$ through the blow-ups

$$
\left\{a_{1}, \ldots, a_{r}\right\} \mapsto\left\{\begin{array}{l}
\left\{1, a_{1}+1, a_{2}, \ldots, a_{r}\right\}, \\
\left\{a_{1}, \ldots, a_{i-2}, a_{i-1}+1,1, a_{i}+1, a_{i+1}, \ldots, a_{r}\right\}, \\
\left\{a_{1}, \ldots, a_{r-1}, a_{r}+1,1\right\} .
\end{array}\right.
$$

We will give an explicit bijection with the triangulation of polygons. A triangulation of a convex polygon $P_{0} P_{1} \ldots P_{r}$ is given by drawing some nonintersecting diagonals on it which divide the polygon into triangles. For a fixed triangulation, we define $v_{i}$ as the number of triangles which have $P_{i}$ as one of their vertices. Note that

$$
\sum_{i=0}^{r} v_{i}=3(r-1)
$$

Using induction, one can show that $\left[a_{1}, \ldots, a_{r}\right]$ is a zero continued fraction if and only if there exists a triangulation of $P_{0} P_{1} \ldots P_{r}$ such that $v_{i}=a_{i}$ for every $1 \leqslant i \leqslant r$. In this way, the number of zero continued fractions of length $r$ is the Catalan number $(1 / r)\left(\begin{array}{c}2(r-1) \\ r-1\end{array}\right)$. Also by induction, one can show that every triangulation has at least two $v_{i}$ equal to 1 . They cannot be adjacent unless $r=2$.

\subsection{Cyclic quotient singularities}

DeFinition 2.2. Given coprime numbers $n$ and $q$ with $0<q<n$, the cyclic quotient singularity $\frac{1}{n}(1, q)$ is the germ at 0 of the quotient of $\mathbb{C}^{2}$ by the action $\zeta \cdot(x, y)=\left(\zeta x, \zeta^{q} y\right)$, where $\zeta$ is a primitive $n$th root of unity.

The minimal resolution of $X=\frac{1}{n}(1, q)$ can be recovered from the continued fraction of $n / q$. If $n / q=\left[e_{1}, \ldots, e_{r}\right]$ with $e_{i} \geqslant 2$ and $\sigma: \tilde{X} \rightarrow X$ is the minimal resolution, the exceptional divisor consists of a chain of $r$ nonsingular rational curves $E_{1}, \ldots, E_{r}$ with $E_{i}^{2}=-e_{i}$. This is pictured in Figure 2.

Note that if we do a blow-up at the intersection of $E_{i}$ and $E_{i+1}$, we get a new chain $E_{1}, \ldots, E_{i}, F, E_{i+1}, E_{r}$ with self-intersections $E_{i}^{2}=-\left(e_{i}+1\right), E_{i+1}^{2}=-\left(e_{i+1}+1\right), F^{2}=-1$. A similar remark can be made for blow-downs. This justifies the terminology blow-down for continued fractions. We note that we can compare the canonical divisors on $X$ and $\tilde{X}$ as follows

$$
K_{\tilde{X}} \equiv \sigma^{*} K_{X}+\sum_{i=1}^{r} k_{i} E_{i}
$$




\section{G. URZÚA AND N. VILCHES}

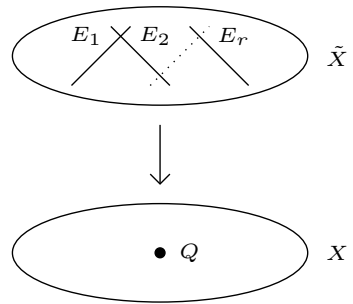

Figure 2. Minimal resolution of $\frac{1}{n}(1, q)$

where $-1<k_{i} \leqslant 0$ are the discrepancies of $E_{i}$.

Definition 2.3. A Wahl singularity is a cyclic quotient singularity $\frac{1}{m^{2}}(1, m a-1)$, where $0<$ $a<m$ and $a$ and $m$ are coprime numbers.

An alternative description can be made by looking at the continued fraction (see [KS88, Lemma 3.11]). Every Wahl singularity arises from [4] by applying the operations

$$
\left[a_{1}, \ldots, a_{r}\right] \mapsto\left\{\begin{array}{l}
{\left[2, a_{1}, \ldots, a_{r-1}, a_{r}+1\right],} \\
{\left[a_{1}+1, a_{2}, \ldots, a_{r}, 2\right] .}
\end{array}\right.
$$

From this algorithm and by induction on $r$, it is clear that every Wahl singularity $m^{2} /(m a-1)=$ $\left[a_{1}, \ldots, a_{r}\right]$ satisfies $\sum_{i=1}^{r} a_{i}=3 r+1$.

Let $\left[a_{1}, \ldots, a_{r}\right]$ be a Wahl continued fraction. We define integers $\delta_{1}, \ldots, \delta_{r}$ in the following inductive way. If $r=1$, then $\delta_{1}:=1$. If we have already defined $\delta_{1}, \ldots, \delta_{r}$ for $\left[a_{1}, \ldots, a_{r}\right]$, then we assign

$$
\begin{array}{lll}
\delta_{1}, \ldots, \delta_{r}, \delta_{1}+\delta_{r} & \text { to } & {\left[a_{1}+1, a_{2}, \ldots, a_{r}, 2\right]} \\
\delta_{1}+\delta_{r}, \delta_{1}, \ldots, \delta_{r} & \text { to } & {\left[2, a_{1}, \ldots, a_{r-1}, a_{r}+1\right] .}
\end{array}
$$

These numbers compute the discrepancies in equation (2.3). If $m^{2} /(m a-1)=\left[a_{1}, \ldots, a_{r}\right]$ has numbers $\delta_{1}, \ldots, \delta_{r}$, then

$$
K_{\tilde{X}} \equiv \sigma^{*} K_{X}+\sum_{i=1}^{r}\left(-1+\frac{\delta_{i}}{\delta_{1}+\delta_{r}}\right) E_{i} .
$$

This gives us explicit control over discrepancies, which will be used to bound them later in this paper.

2.4 Extremal P-resolutions and wormhole singularities. For the study of the components of the deformation space of quotient singularities, Kollár-Shepherd-Barron introduced P-resolutions in [KS88, Section 3]. We only need a particular class of them.

DeFinition 2.4. Let $\Omega$ and $\Delta$ be coprime integers with $0<\Omega<\Delta$, and let $(Q \in Y)$ be a cyclic quotient singularity $\frac{1}{\Delta}(1, \Omega)$. An extremal P-resolution of $(Q \in Y)$ is a partial resolution $f_{0}^{+}:\left(C^{+} \subset X^{+}\right) \rightarrow(Q \in Y)$ such that $X^{+}$has only Wahl singularities, there is one exceptional curve $C^{+}$, which is isomorphic to $\mathbb{P}^{1}$, and $K_{X^{+}}$is relatively ample.

Following [HTU17, Section 4], the surface $X^{+}$has at most two Wahl singularities $\frac{1}{m_{i}^{2}}(1$, $\left.m_{i} a_{i}-1\right)$. If we have smooth points, then we set $m_{i}=a_{i}=1$. If their associated continued 


\section{ON WORMHOLES IN THE MODULI SPACE OF SURFACES}

fractions are given by

$$
\frac{m_{1}^{2}}{m_{1} a_{1}-1}=\left[e_{1}, \ldots, e_{r_{1}}\right], \quad \frac{m_{2}^{2}}{m_{2} a_{2}-1}=\left[f_{1}, \ldots, f_{r_{2}}\right]
$$

and $\left(C^{+}\right)^{2}=-c$ on the minimal resolution of $X^{+}$, then

$$
\frac{\Delta}{\Omega}=\left[f_{r_{2}}, \ldots, f_{1}, c, e_{1}, \ldots, e_{r_{1}}\right] .
$$

We write the extremal P-resolution as $\left[f_{r_{2}}, \ldots, f_{1}\right]-c-\left[e_{1}, \ldots, e_{r_{1}}\right]$. The intersection $K^{+} \cdot C^{+}$ can be computed as $\delta / m_{1} m_{2}$, where $\delta=c m_{1} m_{2}-m_{1} a_{2}-m_{2} a_{1}$. The self-intersection $-c$ of $C^{+}$ can be computed in terms of the continued fraction of $\Delta / \Omega$.

ThEOREM 2.5. Consider a cyclic quotient singularity $Y=\frac{1}{\Delta}(1, \Omega)$, with $\Delta / \Omega=\left[b_{1}, \ldots, b_{r}\right]$. Suppose that we have an extremal P-resolution $\left(C^{+} \subset X^{+}\right)$over $\frac{1}{\Delta}(1, \Omega)$ with $l$ singularities $(l=0,1$ or 2$)$. Then, the self-intersection of the exceptional curve $C^{+}$on the minimal resolution of $X^{+}$is $-\left(\sum_{i=1}^{r} b_{i}-3 r+3-l\right)$.

As a direct consequence, note that if $\sum_{i=1}^{r} b_{i}<3 r$, there are no extremal P-resolutions. If $\sum_{i=1}^{r} b_{i}=3 r$, then $c$ can be -1 (if there are two singularities) or -2 (if there is only one singularity) or -3 (if $l=0$ ), and so on.

Proof. If $l=0$, then $r=1$ and the result is trivially true. Suppose $l=2$; the proof for $l=1$ is similar. Consider the extremal P-resolution

$$
\left[f_{r_{2}}, \ldots, f_{1}\right]-c-\left[e_{1}, \ldots, e_{r_{1}}\right] .
$$

Note that $\sum_{i=1}^{r_{1}} e_{i}+c+\sum_{j=1}^{r_{2}} f_{j}=3\left(r_{1}+r_{2}\right)+c+2$ since we have two Wahl singularities, and so $\sum_{i=1}^{r_{1}} e_{i}=3 r_{1}+1$ and $\sum_{j=1}^{r_{2}} f_{j}=3 r_{2}+1$.

From [KS88, Lemmas 3.13 and 3.14], we know that, from the minimal resolution of $Y$, one has to blow up only at the intersection points of exceptional curves to obtain the minimal resolution of the extremal P-resolution. In this way, the sum of the self-intersections of the exceptional curves plus three times their number remains constant at each blow-up (since at every blow-up, we subtract 3 from the sum of the self-intersections, and we add 1 to the number of curves). This shows that

$$
\sum_{i=1}^{r} b_{i}-3 r=\left(\sum_{i=1}^{r_{1}} e_{i}+c+\sum_{j=1}^{r_{2}} f_{j}\right)-3\left(r_{1}+r_{2}+1\right) .
$$

It follows that $\sum_{i=1}^{r} b_{i}-3 r=c-1$.

Given a coprime pair $\Omega, \Delta$ with $0<\Omega<\Delta$, one can find all possible extremal P-resolutions by looking at the dual fraction $\Delta /(\Delta-\Omega)$. More precisely, we have the following result (see [HTU17, Proposition 4.1]).

Proposition 2.6. If $\Delta /(\Delta-\Omega)=\left[c_{1}, \ldots, c_{s}\right]$, then there is a bijection between extremal $P$ resolutions and pairs $1 \leqslant \alpha<\beta \leqslant s$ such that

$$
\left[c_{1}, \ldots, c_{\alpha-1}, c_{\alpha}-1, c_{\alpha+1}, \ldots, c_{\beta-1}, c_{\beta}-1, c_{\beta+1}, \ldots, c_{s}\right]=0 .
$$

Moreover, the $a_{i}, m_{i}$, and $\delta$ of the corresponding extremal P-resolution (see right after Definition 2.4) can be computed as $m_{2} / a_{2}=\left[c_{1}, \ldots, c_{\alpha-1}\right], m_{1} / a_{1}=\left[c_{s}, \ldots, c_{\beta+1}\right]$ (if $\alpha=1$ or $\beta=s$, the associated points are smooth), and $\delta / \varepsilon=\left[c_{\alpha+1}, \ldots, c_{\beta-1}\right]$, where $0<\varepsilon<\delta$ (or $\delta=1$ if $\alpha+1=\beta)$. 


\section{G. URzÚa AND N. VilcheS}

It will be useful to denote the expression in equation (2.6) with two bars as

$$
\left[c_{1}, \ldots, \bar{c}_{\alpha}, \ldots, \bar{c}_{\beta}, \ldots, c_{s}\right] \text {. }
$$

Moreover, if it admits a second extremal P-resolution, then we will denote it with two underlines. For instance, if $\Delta=36$ and $\Omega=13$, then we write

$$
\frac{36}{36-13}=[2, \underline{3}, \overline{2}, \underline{2}, \overline{4}]
$$

and so we know that it admits two extremal P-resolutions, and we know how to obtain them. In this example, $[2,3, \overline{2}, 2, \overline{4}]$ is associated with the extremal P-resolution $[3,5,2]-2$, and $[2, \overline{3}, 2, \overline{2}, 4]$ corresponds to $[4]-1-[6,2,2]$.

Definition 2.7. As in [HTU17, Section 4], a sequence $\left\{a_{1}, \ldots, a_{r}\right\}$ with $a_{i}>1$ is of $W W$ type if there exist $\alpha$ and $\beta$ with $1 \leqslant \alpha<\beta \leqslant r$ such that

$$
\left[a_{1}, \ldots, \bar{a}_{\alpha}, \ldots, \bar{a}_{\beta}, \ldots, a_{r}\right]=0 .
$$

A wormhole singularity is a cyclic quotient singularity $\frac{1}{\Delta}(1, \Omega)$ which admits at least two extremal P-resolutions. Equivalently, the continued fraction of $\Delta /(\Delta-\Omega)$ is of WW type by means of at least two pairs $(\alpha, \beta)$ and $\left(\alpha^{\prime}, \beta^{\prime}\right)$.

As a consequence of Theorem 2.8 below, a wormhole singularity admits precisely two extremal P-resolutions.

If $\left\{a_{1}, \ldots, a_{r}\right\}$ is a sequence of WW type, there is a triangulation of a polygon $P_{0} P_{1} \ldots P_{r}$ such that $v_{i}=a_{i}$. Thus, we define $a_{0}:=v_{0}$. Note that by equation $(2.2)$, we have $a_{0}=3 r-1-\sum_{i=1}^{r} a_{i}$, so it does not depend on the pair $(\alpha, \beta)$. Also note that $a_{0}$ may be 1 .

Therefore, we have two cases: (A) $a_{0}>1$ or (B) $a_{0}=1$ (as in [HTU17, Section 4.2]). We will focus on proving statements for case (A) since from that proof we will deduce case (B) as a consequence. The main idea is that we can "remove" the vertex $P_{0}$ from the polygon $P_{0} P_{1} \ldots P_{r}$ and repeat this action until all entries are greater than 1.

Our next goal is to give a simplified proof of [HTU17, Theorem 4.3] and a new proof of [HTU17, Theorem 4.4].

TheOREM 2.8 ([HTU17, Theorem 4.3]). A cyclic quotient singularity has at most two distinct extremal P-resolutions.

TheOREM 2.9 ([HTU17, Theorem 4.4]). If a cyclic quotient singularity admits two extremal $P$-resolutions, then the values of $\delta$ are equal.

To prove Theorem 2.8, note that by Proposition 2.6, it suffices to show that a sequence of WW type $\left\{a_{1}, \ldots, a_{r}\right\}$ admits at most two pairs $(\alpha, \beta)$ such that

$$
\left[a_{1}, \ldots, a_{\alpha}-1, \ldots, a_{\beta}-1, \ldots, a_{r}\right]=0 .
$$

Let $a_{0}=3 r-1-\sum_{i=1}^{r} a_{i}$ as before, and assume that we are in case (A), that is, $a_{0}>1$. Since the triangulation of $P_{0} P_{1} \ldots P_{r}$ needs to have at least two vertices with $v_{i}=1$, we must have $a_{\alpha}-1=a_{\beta}-1=1$, and thus $a_{\alpha}=a_{\beta}=2$.

Then note that $\left[a_{\alpha+1}, \ldots, a_{\beta}-1, \ldots, a_{r}, a_{0}, a_{1}, \ldots, a_{\alpha-1}\right]=0$ since we have a triangulation. A matrix computation shows that

$$
\frac{m}{m-a}=\left[a_{\beta-1}, \ldots, a_{\alpha+1}\right], \quad \frac{m^{\prime}}{m^{\prime}-a^{\prime}}=\left[a_{\beta+1}, \ldots, a_{r}, a_{0}, \ldots, a_{\alpha-1}\right]
$$




\section{ON WORMHOLES IN THE MODULI SPACE OF SURFACES}

has $m=m^{\prime}$ and $a+a^{\prime}=m$. In this way, we see that

$$
\left[a_{\alpha+1}, \ldots, a_{r}, a_{0}, \ldots, a_{\alpha-1}\right]=\frac{m^{2}}{m a+1}
$$

is the dual of a Wahl singularity. All of them are obtained from $[2,2,2]$ by applying the same procedure from equation (2.4), which can be seen as a consequence of Riemenschneider diagrams. See [HTU17, Section 4.2] for another proof of this fact.

Thus, to produce sequences of WW type, we start with $[2, \overline{2}, 2]$, then we apply equation $(2.4)$, and finally we add a $\overline{2}$ to close the "cycle." After that, we choose one of the entries different from $\overline{2}$ and remove it. That entry will be the $a_{0}$.

To simplify the proof of Theorem 2.8, we will use the following sequences of 0 s and $1 \mathrm{~s}$.

Definition 2.10. Given $\left\{a_{0}, \ldots, a_{r}\right\}, a_{i} \geqslant 2$, we define its indicator sequence as

$$
\{1, \underbrace{0, \ldots, 0}_{a_{0}-2}, 1, \underbrace{0, \ldots, 0}_{a_{1}-2}, 1, \ldots, 1, \underbrace{0, \ldots, 0}_{a_{r}-2}\} .
$$

We consider $\left\{a_{0}, \ldots, a_{r}\right\}$ and its indicator sequence indexed by a cyclic group. As an example, the indicator sequence of $\{2,3,4,2,3\}$ is $\{1,1,0,1,0,0,1,1,0\}$. Note that we can completely recover the sequence $\left\{a_{0}, \ldots, a_{r}\right\}$ from the indicator sequence, and so we can study sequences of WW type from their indicator sequences in case (A). We also note that, for every $i$, there are two indices $l_{i}$ and $m_{i}$ such that $e_{l_{i}+1}, \ldots, e_{m_{i}-1}$ are all the zeroes induced by $a_{i}$. The main advantage of this is that it makes the procedure from equation (2.4) more symmetric. We start with $\overline{1,1}$; then, we add 1 to one side and 0 to the other, as follows

$$
\{\overline{1,1}\} \rightarrow\{0, \overline{1,1}, 1\} \rightarrow\{1,0, \overline{1,1}, 1,0\} \rightarrow\{1,1,0, \overline{1,1}, 1,0,0\} \rightarrow \cdots .
$$

We repeat and then add $\overline{1,1}$ to the end. In particular, all these indicator sequences have an even number of entries, and the numbers 1 which corresponds to the $\overline{2}$ are opposites.

Proof of Theorem 2.8. We assume $a_{0}>1$ as before. We consider a sequence of WW type $\left\{a_{1}, \ldots, a_{r}\right\}$ and its indicator sequence $\left\{e_{0}, \ldots, e_{2 m-1}\right\}$. Consider $p$ and $q$ with $p<q$ such that $\left[a_{1}, \ldots, \bar{a}_{p}, \ldots, \bar{a}_{q}, \ldots, a_{r}\right]=0$. Thus, if $t, t+1$ and $t+m, t+m+1$ are the corresponding indices for $a_{p}=2$ and $a_{q}=2$, the construction yields

$$
e_{j}= \begin{cases}2-e_{2 t+1-j}, & j=r, r+1, r+m, r+m+1 ; \\ 1-e_{2 t+1-j}, & j \neq r, r+1, r+m, r+m+1 .\end{cases}
$$

Given the indicator sequence, it then suffices to show that there are at most two pairs $\{t, t+m\}$ which makes equation (2.7) true. Since $t$ and $t+m$ give the same pair, we will define $f_{j}=$ $\left(e_{j}+e_{j+m}\right) / 2$ as a sequence indexed by $\mathbb{Z} / m \mathbb{Z}$. Equation $(2.7)$ translates to

$$
f_{j}= \begin{cases}2-f_{2 t+1-j}, & j=r, r+1 ; \\ 1-f_{2 t+1-j}, & j \neq r, r+1 .\end{cases}
$$

We are going to use the same trick as in [HTU17, Section 4.2]. Fix a primitive $m$ th root of unity $\mu$, and define

$$
F=\sum_{j=0}^{m-1} \mu^{j} f_{j}
$$




\section{G. URzÚa AND N. VilcheS}

Adding equation (2.8) multiplied by $\mu^{j}$ for $j=0$ to $m$, we get

$$
\left(\mu^{t}\right)^{2} \cdot \mu \bar{F}-\left(\mu^{t}\right) \cdot(\mu+1)+F=0 .
$$

For $m>2$, this is an equation of degree at least 1 in $\mu^{t}$. Thus, there are at most two valid values of $\mu^{t}$. Note that $m=2$ happens only for the indicator sequence $\{1,1,1,1\}$ associated with $\{\overline{2}, \underline{2}, \overline{2}, \underline{2}\}$. By the correspondence between sequences of type WW in case (A) and indicator sequences, this shows that there are at most two pairs in this case.

Now suppose $a_{0}=1$; that is, we are now in case $(\mathrm{B})$. Note that for every pair $(\alpha, \beta)$ with $\alpha<\beta$ such that $\left[a_{1}, \ldots, \bar{a}_{\alpha}, \ldots, \bar{a}_{\beta}, \ldots, a_{r}\right]=0$, the corresponding triangulation on $P_{0} P_{1} \ldots P_{r}$ must have a triangle $P_{0} P_{1} P_{r}$. We can then remove vertex $P_{0}$ and look at pairs for the new sequence $a_{1}-1, a_{2}, \ldots, a_{r-1}, a_{r}-1$ since it is easy to show that they are in bijection with pairs for the original sequence. Inductively, this reduces case (B) to case (A).

Proof of Theorem 2.9. We will use Proposition 2.6. Consider a sequence $\left\{a_{1}, \ldots, a_{r}\right\}$ with $a_{0}>1$; that is, we are in case (A). If $(p, q)$ is a pair with $p<q$ such that $\left[a_{1}, \ldots, \bar{a}_{p}, \ldots, \bar{a}_{q}, \ldots, a_{r}\right]=0$, then $\left[a_{p+1}, \ldots, a_{q-1}\right]=\delta / \varepsilon$ for some $\varepsilon$. Thus,

$$
\left[a_{p+1}, \ldots, a_{r}, a_{0}, \ldots, a_{p-1}\right]=\frac{\delta^{2}}{\delta \lambda+1}
$$

for some $\lambda<\delta$. Since all entries are at least 2, we can compute

$$
\left(\begin{array}{cc}
a_{p+1} & -1 \\
1 & 0
\end{array}\right) \cdots\left(\begin{array}{cc}
a_{r} & -1 \\
1 & 0
\end{array}\right)\left(\begin{array}{cc}
a_{0} & -1 \\
1 & 0
\end{array}\right) \cdots\left(\begin{array}{cc}
a_{p-1} & -1 \\
1 & 0
\end{array}\right)=\left(\begin{array}{cc}
\delta^{2} & -\delta(\delta-\lambda)-1 \\
\delta \lambda+1 & -\lambda(\delta-\lambda)-1
\end{array}\right) .
$$

Since $a_{p}=2$, we obtain that the matrix

$$
\left(\begin{array}{cc}
a_{p+1} & -1 \\
1 & 0
\end{array}\right) \cdots\left(\begin{array}{cc}
a_{r} & -1 \\
1 & 0
\end{array}\right)\left(\begin{array}{cc}
a_{0} & -1 \\
1 & 0
\end{array}\right) \cdots\left(\begin{array}{cc}
a_{p-1} & -1 \\
1 & 0
\end{array}\right)\left(\begin{array}{cc}
a_{p} & -1 \\
1 & 0
\end{array}\right)
$$

is

$$
\left(\begin{array}{cc}
\delta_{i}^{2}+\delta_{j} \lambda_{i}-1 & -\delta_{i}^{2} \\
\delta_{i} \lambda_{i}+\lambda_{i}^{2}+1 & -\delta_{i} \lambda_{i}-1
\end{array}\right)
$$

Its trace is exactly $\delta^{2}-2$. But recall that the trace of a multiplication is invariant under cyclic permutations of the factors, which shows that

$$
\operatorname{tr}\left(\left(\begin{array}{cc}
a_{0} & -1 \\
1 & 0
\end{array}\right) \ldots\left(\begin{array}{cc}
a_{r} & -1 \\
1 & 0
\end{array}\right)\right)=\delta^{2}-2 .
$$

The left-hand side does not depend on the pair $(p, q)$; thus $\delta$ is the same for every pair. This proves Theorem 2.9 for case (A).

Case (B) is handled by induction to reduce it to case (A), just as in the proof of Theorem 2.8. If $a_{0}=1$, we can blow down the sequence there. Note that

$$
\left(\begin{array}{cc}
a_{r} & -1 \\
1 & 0
\end{array}\right)\left(\begin{array}{cc}
1 & -1 \\
1 & 0
\end{array}\right)\left(\begin{array}{cc}
a_{1} & -1 \\
1 & 0
\end{array}\right)=\left(\begin{array}{cc}
a_{r}-1 & -1 \\
1 & 0
\end{array}\right)\left(\begin{array}{cc}
a_{1}-1 & -1 \\
1 & 0
\end{array}\right)
$$

which shows that the trace remains constant. Also, since the interval $a_{p+1}, \ldots, a_{q-1}$ is not affected by blow-downs, this inductively reduces it to case $(\mathrm{A})$. 


\section{ON WORMHOLES IN THE MODULI SPACE OF SURFACES}

\section{General setup and the wormhole conjecture}

In this section, we will look at singular surfaces together with a smoothing over a smooth analytic curve germ $\mathbb{D}$. This point of view was used in [Urz16b] under the name of $\mathrm{W}$-surfaces, and it works better for the setup of the wormhole conjecture. We start by recalling it.

\subsection{W-surfaces and their MMP}

Definition 3.1. A $W$-surface is a normal projective surface $X$ together with a proper deformation $(X \subset \mathcal{X}) \rightarrow(0 \in \mathbb{D})$ such that

(i) $X$ has at most Wahl singularities,

(ii) $\mathcal{X}$ is a normal complex 3 -fold with $K_{\mathcal{X}} \mathbb{Q}$-Cartier,

(iii) the fiber $X_{0}$ is reduced and isomorphic to $X$,

(iv) the fiber $X_{t}$ is nonsingular for $t \neq 0$.

A W-surface is said to be smooth if $X$ is nonsingular.

Various basic properties of $\mathrm{W}$-surfaces are shown in [Urz16b, Section 2]. A W-surface $X$ is minimal if $K_{X}$ is nef. This is equivalent to $K_{\mathcal{X}}$ being nef, as it is shown in [Urz16b, Lemma 2.3]. If a $\mathrm{W}$-surface $X$ is not minimal, then there is an explicit MMP relative to $\mathbb{D}$ which we will review briefly below. The outcomes of this MMP are discussed in [Urz16b, Section 2]. We note that invariants such as irregularity, geometric genus, $K^{2}$, and topological Euler characteristic are constant for the fibers in a W-surface. An invariant that may not remain constant is the topological fundamental group. We have that $K_{X}$ ample implies $K_{X_{t}}$ ample for all $t$, and in this case we may think of a W-surface $X$ as a disk in the KSBA compactification of the moduli space of surfaces of general type with $K^{2}=K_{X}^{2}$ and $\chi=\chi\left(\mathcal{O}_{X}\right)$.

Let $\sigma: \widetilde{X} \rightarrow X$ be the minimal resolution of $X$.

Lemma 3.2. Let $X$ be a minimal $W$-surface such that the minimal resolution of $X$ is ruled. Then $X$ is rational.

Proof. Assume that $\widetilde{X}$ is ruled but not rational. Then there is a fibration $\widetilde{X} \rightarrow C$ with general fiber $\mathbb{P}^{1}$ and $C$ a nonsingular projective curve of positive genus. Then all curves in the exceptional divisor of $\sigma$ must be contained in fibers. But if $F$ is a general fiber, then $F \cdot K_{\tilde{X}}=\sigma(F) \cdot K_{X}$ and, by adjunction, $F \cdot K_{\tilde{X}}=-2$, which is contrary to the assumption that $K_{X}$ is nef.

When a W-surface $X$ has $K_{X}$ not nef, there is a smooth rational curve $C$ with $C \cdot K_{X}<0$. The cases $C^{2} \geqslant 0$ are analyzed in [Urz16b, Section 2]; these are not relevant to the present paper. We assume $C^{2}<0$. Then the $\mathrm{W}$-surface $X$ defines an extremal neighborhood of type mk1A or mk2A, and we need to run the MMP on the 3-fold family $(X \subset \mathcal{X}) \rightarrow(0 \in \mathbb{D})$. Roughly speaking, in case of a flip, we will replace $C$ with a $K$-positive curve $C^{+} \subset X^{+}$, obtaining a new family $\left(X^{+} \subset \mathcal{X}^{+}\right) \rightarrow(0 \in \mathbb{D})$, where fibers over $t \neq 0$ remain equal to the fibers of the first family. In this way, the surface $X^{+}$defines a new $\mathrm{W}$-surface. In case of a divisorial contraction, we will have a divisor in $\mathcal{X}$ whose restriction to $X$ is $C$ and whose restriction to any other fiber is a $(-1)$-curve. The contraction of this divisor gives us a new family, and the contraction of $C$ produces a Wahl singularity. The new surface is a W-surface. For details, we refer to [Urz16a, Section 2.4] (see also [HTU17, Section 2], [Urz16b, Section 2]). Below, we describe the mk1A and mk2A situations on the surface $X$. Let $(C \subset X) \rightarrow(Q \in Y)$ be the contraction of $C$. 


\section{G. URzÚa AND N. VilcheS}

Type $m k 1 A$. In this case, $X$ has one Wahl singularity $\frac{1}{m^{2}}(1, m a-1)$, where $m^{2} /(m a-1)=$ $\left[e_{1}, \ldots, e_{s}\right]$. Let $E_{1}, \ldots, E_{s}$ be the corresponding exceptional curves in $\widetilde{X}$, so that $E_{j}^{2}=-e_{j}$. The proper transform $\tilde{C}$ of $C$ is a smooth rational curve intersecting only one $E_{i}$ transversally at one point. The curve $C$ contracts to $(Q \in Y)$, which is the cyclic quotient singularity $\frac{1}{\Delta}(1, \Omega)$, where

$$
\frac{\Delta}{\Omega}=\left[e_{1}, \ldots, e_{i-1}, e_{i}-1, e_{i+1}, \ldots, e_{s}\right]
$$

We will denote this situation by $\left[e_{1}, \ldots, \overline{e_{i}}, \ldots, e_{s}\right]$. If we write $K_{\widetilde{X}} \equiv \sigma^{*}\left(K_{X}\right)+\sum_{j=1}^{s}(-1+$ $\left.\left(\delta_{j} / m\right)\right) E_{j}$ and $\delta:=\delta_{i}$, we have

$$
\tilde{C} \cdot K_{\tilde{X}}=-1+\frac{\delta}{m}+C \cdot K_{X}<C \cdot K_{X}<0,
$$

and $\tilde{C}^{2}<0$ since it is contracted. In particular, the curve $\tilde{C}$ is a $(-1)$-curve. We have $C \cdot K_{X}=$ $-\delta / m$ and $C^{2}=-\Delta / m^{2}$.

Type mk2A. In this case, $X$ has two Wahl singularities $\frac{1}{m_{j}^{2}}\left(1, m_{j} a_{j}-1\right)$ for $j=1$, 2 , where $m_{1}^{2} /\left(m_{1} a_{1}-1\right)=\left[e_{1}, \ldots, e_{s_{1}}\right]$ and $m_{2}^{2} /\left(m_{2} a_{2}-1\right)=\left[f_{1}, \ldots, f_{s_{2}}\right]$. Let $E_{1}, \ldots, E_{s_{1}}$ and $F_{1}, \ldots, F_{s_{2}}$ be the corresponding exceptional curves with $E_{j}^{2}=-e_{j}$ and $F_{j}^{2}=-f_{j}$. The strict transform $\tilde{C}$ of $C$ is a smooth rational curve intersecting only $E_{1}$ and $F_{1}$, transversally at one point each. We have that

$$
\frac{\Delta}{\Omega}=\left[f_{s_{2}}, \ldots, f_{1}, 1, e_{1}, \ldots, e_{s_{1}}\right],
$$

where $(Q \in Y)$ is $\frac{1}{\Delta}(1, \Omega)$. Let $\delta:=m_{2} a_{1}-m_{1}\left(m_{2}-a_{2}\right)$. Then we have

$$
\tilde{C} \cdot K_{\tilde{X}}=-1+\frac{\delta}{m_{1} m_{2}}+C \cdot K_{X}<C \cdot K_{X}<0
$$

and we know $\tilde{C}^{2}<0$. In particular, the curve $\tilde{C}$ is a $(-1)$-curve. We have $C \cdot K_{X}=-\delta / m_{1} m_{2}$ and $C^{2}=-\Delta /\left(m_{1}^{2} m_{2}^{2}\right)$.

To know whether a W-surface $X$ with $C \cdot K_{X}<0$ and $C^{2}<0$ defines a flip or divisorial contraction, we need to run Mori's algorithm from the numerical data of the mk1A or mk2A extremal neighborhood. We refer to [Urz16a, Section 2.4] for details; see also [Vil20] for examples and computer implementation of Mori's algorithm. A summary with relevant properties for us is the following.

Divisorial contraction. In this case, the general fiber of the $\mathrm{W}$-surface $X$ contains a (-1)-curve which deforms to $C$. This gives us a divisor on the 3 -fold $\mathcal{X}$, which can be contracted to obtain a new W-surface $Y$. The contraction of $C \subset X$ produces a Wahl singularity $(Q \in Y)$.

Flip. In this case, the contraction of $C$ produces a cyclic quotient singularity $\frac{1}{\Delta}(1, \Omega)=(Q \in Y)$. This singularity admits an extremal P-resolution $\left(C^{+} \subset X^{+}\right) \rightarrow(Q \in Y)$ so that a suitable Wsurface $X^{+}$is the flip of the $\mathrm{W}$-surface $X$. The general fibers of the $\mathrm{W}$-surfaces $X$ and $X^{+}$are isomorphic.

If a multiple of $K_{X}$ has sections, then after finitely many flips and/or divisorial contractions of type mk1A and/or mk2A, we will obtain a minimal W-surface (see, for example, [HTU17, Theorem 5.3]). Otherwise, after finitely many flips and/or divisorial contractions of type mk1A and/or mk2A, we will end up with either a smooth deformation of a ruled surface, or a degeneration of $\mathbb{P}^{2}$ (see, for example, [Urz16b]). 


\section{ON WORMHOLES IN THE MODULI SPACE OF SURFACES}

3.2 Wormholes. The following is the setup for a wormhole. We take a W-surface $X_{1}$ with $K_{X_{1}}$ ample, and we assume that $X_{1}$ has an extremal P-resolution $\left(C_{1} \subset X_{1}\right) \rightarrow(Q \in Y)$ over a WW singularity $(Q \in Y)$. In this way, the surface $Y$ is obtained from $X_{1}$ by contracting one smooth rational curve. As cyclic quotient singularities are rational, the irregularity and geometric genus of both surfaces are equal. By the Nakai-Moishezon criterion, the surface $Y$ is a KSBA stable surface, but it does not belong to the same moduli space as $X_{1}$ since $K_{Y}^{2}=K_{X_{1}}^{2}-\nu^{2} C_{1}^{2}$ for some $\nu \neq 0$ and $C_{1}^{2}<0$ (as it is contracted).

Let $E$ be the exceptional (reduced) divisor of the minimal resolution $\widetilde{X_{1}} \rightarrow X_{1}$, and let $\widetilde{C_{1}}$ be the strict transform of $C_{1}$. We also assume

$$
H^{2}\left(\widetilde{X_{1}}, T_{\widetilde{X_{1}}}^{0}\left(-\log \left(E+\widetilde{C_{1}}\right)\right)\right)=0 .
$$

By [LP07, Section 2], this condition can be used to prove that there are no local-to-global obstructions to deform $X_{1}$ (which, in particular, shows the existence of W-surfaces $X_{1}$ ). If $\widetilde{C_{1}}$ is a $(-1)$-curve, then $H^{2}\left(\widetilde{X_{1}}, T_{\widetilde{X_{1}}}^{0}\left(-\log \left(E+\widetilde{C_{1}}\right)\right)\right)=0$ is the same as

$$
H^{2}\left(\widetilde{X_{1}}, T_{\widetilde{X_{1}}}^{0}(-\log (E))\right)=0,
$$

and this is the same as requiring $H^{2}\left(X_{1}, T_{X_{1}}^{0}\right)=0$ by [LP07, Theorem 2]. Let $X_{2}$ be the surface resulting by contracting the extremal P-resolution in $X_{1}$ and then partially resolving with the second extremal P-resolution of $(Q \in Y)$. Hence the surface $Y$ is the contraction of a smooth rational curve $C_{2}$ in $X_{2}$. So far, we have that $Y$ lives in a different moduli space than $X_{1}$ and $X_{2}$, but it is not clear whether $X_{1}$ and $X_{2}$ (or its KSBA replacement) live in the same moduli space.

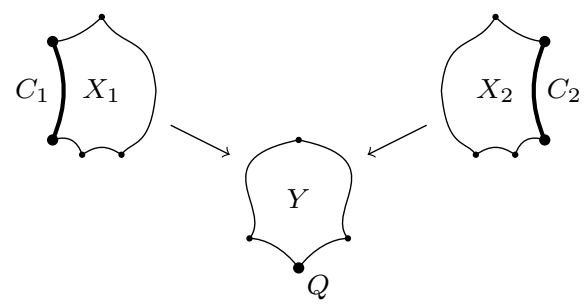

FiguRE 3. The three singular surfaces in a wormhole

Lemma 3.3. We have that $X_{2}$ defines a $W$-surface, and $K_{X_{1}}^{2}=K_{X_{2}}^{2}$ and $\chi\left(\mathcal{O}_{X_{1}}\right)=\chi\left(\mathcal{O}_{X_{2}}\right)$.

Proof. We need to prove the existence of a $\mathbb{Q}$-Gorenstein smoothing for $X_{2}$. We know that

$$
H^{2}\left(\widetilde{X_{1}}, T_{\widetilde{X_{1}}}^{0}\left(-\log \left(E+\widetilde{C_{1}}\right)\right)\right)=0 .
$$

Let $A_{1}$ be the chain formed by the exceptional curves of the extremal P-resolution and $\widetilde{C_{1}}$. Let $A_{2}$ be the chain formed by the exceptional curves of the second extremal P-resolution together with the corresponding curve $\widetilde{C_{2}}$. We know that to obtain $A_{2}$, we perform blow-downs until reaching the exceptional chain of $(Q \in Y)$, and then we perform blow-ups at that chain to obtain $A_{2}$. (We may not need blow-downs and/or blow-ups, of course.) By the addition/deletion principle of (-1)-curves (see, for example, [LP07, Proposition 6]) applied at each blow-down and blow-up, we have

$$
H^{2}\left(\widetilde{X_{1}}, T_{\widetilde{X}_{1}}^{0}\left(-\log \left(E+\widetilde{C_{1}}\right)\right)\right)=H^{2}\left(\widetilde{X_{2}}, T_{\widetilde{X}_{2}}^{0}\left(-\log \left(E^{\prime}+\widetilde{C_{2}}\right)\right)\right)
$$

where $E^{\prime}$ is the exceptional divisor of the minimal resolution $\widetilde{X_{2}} \rightarrow X_{2}$. Therefore, by our hypothesis, we have $H^{2}\left(\widetilde{X}_{2}, T_{\widetilde{X}_{2}}^{0}\left(-\log \left(E^{\prime}+\widetilde{C}_{2}\right)\right)\right)=0$. By using the standard short exact 


\section{G. URzÚa AND N. VilcheS}

sequence

$$
0 \rightarrow T_{\widetilde{X_{2}}}^{0}\left(-\log \left(E^{\prime}+\widetilde{C_{2}}\right)\right) \rightarrow T_{\widetilde{X_{2}}}^{0}\left(-\log \left(E^{\prime}\right)\right) \rightarrow \mathcal{N}_{\widetilde{C_{2}} / \widetilde{X_{2}}} \rightarrow 0
$$

we see that $H^{2}\left(\widetilde{X_{2}}, T_{\widetilde{X}_{2}}^{0}\left(-\log \left(E^{\prime}\right)\right)\right)=0$. Hence, by [LP07, Theorem 2], there are no local-toglobal obstructions to deform $X_{2}$, and so we have a W-surface $X_{2}$.

In relation to invariants, since Wahl singularities are rational, we clearly have $\chi\left(\mathcal{O}_{X_{1}}\right)=$ $\chi\left(\mathcal{O}_{X_{2}}\right)$. As for $K^{2}$, we note that if $X$ is a normal projective surface with only Wahl singularities and $\widetilde{X} \rightarrow X$ is the minimal resolution, then $K_{X}^{2}=K_{\widetilde{X}}^{2}+l$, where $l$ is the number of exceptional curves. As $A_{2}$ is obtained by blow-downs and blow-ups on $A_{1}$ and we contract all curves except one, we obtain that $K_{X_{1}}^{2}=K_{X_{2}}^{2}$.

Therefore, we have a $\mathrm{W}$-surface $X_{2}$ with the same invariants as $X_{1}$. However, $K_{X_{2}}$ may not be nef.

Conjecture 3.4 (Wormhole conjecture). The MMP on the new W-surface finishes in a minimal model, and it requires only flips; that is, both punctured W-surfaces live in the same moduli space.

A main purpose of this paper is to show that Conjecture 3.4 is true when $X_{1}$ is not rational and for a wide range of WW singularities. One may hope that perhaps in the case when $X_{1}$ is not rational, we do have that $K_{X_{2}}$ is nef. We will prove that this is true in many situations, but the following example shows that it is not always the case.

Example 3.5. We consider an Enriques surface with the configuration of $(-2)$-curves shown in Figure 4. This configuration is proved to exist in [DRU20, Section 2.2].

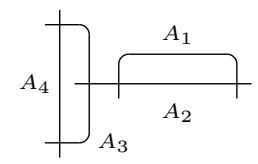

FiguRE 4. Special curves in an Enriques surface

We do five blow-ups to get the configuration in Figure 5 . The exceptional curves $E_{1}, \ldots, E_{5}$ are indexed according to the order of the blow-ups.

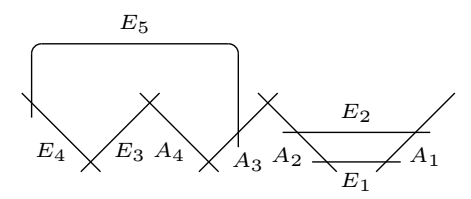

Figure 5. After five blow-ups

We have $E_{1}^{2}=E_{2}^{2}=E_{5}^{2}=-1, E_{3}^{2}=E_{4}^{2}=-2, A_{4}^{2}=-3, A_{1}^{2}=A_{2}^{2}=-4, A_{3}^{2}=-5$. After contracting $E_{1}$, the chain of curves

$$
E_{4}-E_{3}-A_{4}-A_{3}-A_{2}-E_{1}-A_{1}
$$

corresponds to the minimal resolution of the singularity $\frac{1}{235}(1,169)$ since $[2,2,3,5,3,3]=235 / 169$. This is a WW singularity, which define surfaces $X_{1}$ and $X_{2}$. In both cases, we have W-surfaces $X_{1}$ and $X_{2}$ because we can prove that they do not have obstructions (see [DRU20, Lemma 2.4]). 
If we contract $A_{1}$ and $A_{2}-A_{3}-A_{4}-E_{3}-E_{4}$ to singularties $P_{1}$ and $P_{2}$, then we obtain the surface $X_{1}$ with the extremal P-resolution $[2,2,3,5,4]-1-[4]$. It can be proved that a general $X_{1}$ has $K_{X_{1}}$ ample.

If we contract $E_{3}-A_{4}-A_{3}-A_{2}-A_{1}-E_{1}$ to a point $P_{3}$, then we get the surface $X_{2}$ with the extremal P-resolution $2-[2,3,5,3,3]$. But in this case, we have $K \cdot E_{5}=-1 / 13$. The curve $E_{5}$ induces an mk1A neighborhood. The numerical data for this $\mathrm{mk} 1 \mathrm{~A}$ is $[2,3,5-1,3,3]=129 / 79$, which is not a Wahl singularity, and so this is a flipping mk1A. The extremal P-resolution which does the flip is $[2,3,5,3]-1-[2,5,3]$. This is in Figure 6 , where $F_{1}, F_{2}, F_{3}$ are the new curves from the new blow-ups. We note that $E_{2}^{2}=E_{4}^{2}=-1$, and these are the only curves that could be negative for the canonical divisor $K$ of the new singular surface.

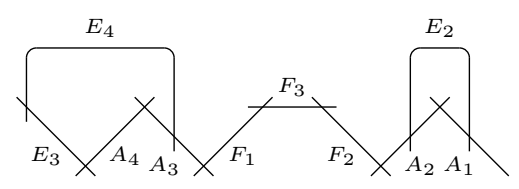

Figure 6. After the flip

However, we compute $K \cdot E_{4}=\frac{1}{4}$ and $K \cdot E_{2}=\frac{3}{5}$. This proves that $K$ is now nef. We only used one flip to obtain the nef model, and we have a wormhole in the moduli space of $\mathbb{Z} / 2$-Godeaux surfaces.

We now prove a relevant reduction step toward Conjecture 3.4. Let us consider W-surfaces $X_{1}$ and $X_{2}$ as in Conjecture 3.4. Let $\left(X_{1} \subset \mathcal{X}_{1}^{\prime}\right) \rightarrow(0 \in \mathbb{D})$ be a partial $\mathbb{Q}$-Gorenstein deformation which keeps the distinguished extremal P-resolution in all fibers but smooths all other Wahl singularities. This is possible since we have $H^{2}\left(\widetilde{X_{1}}, T_{\widetilde{X}_{1}}^{0}\left(-\log \left(E+\widetilde{C_{1}}\right)\right)\right)=0$. We denote the general fiber by $X_{1}^{\prime}$. Let $\left(X_{2} \subset \mathcal{X}_{2}^{\prime}\right) \rightarrow(0 \in \mathbb{D})$ be the $\mathbb{Q}$-Gorenstein deformation obtained by first contracting the extremal P-resolution of all fibers in $\left(X_{1}^{\prime} \subset \mathcal{X}_{1}^{\prime}\right) \rightarrow(0 \in \mathbb{D})$ (where this deformation is trivial) and then gluing the other extremal P-resolution. The general fiber is denoted by $X_{2}^{\prime}$. Since we do not have local-to-global obstructions, there are W-surfaces $X_{1}^{\prime}$ and $X_{2}^{\prime}$ as in the setup of Conjecture 3.4.

Lemma 3.6. If Conjecture 3.4 is true for the $W$-surfaces $X_{1}^{\prime}$ and $X_{2}^{\prime}$, then it is also true for the $W$-surfaces $X_{1}$ and $X_{2}$.

Proof. The point is that the $\mathbb{Q}$-Gorenstein deformation space of the surfaces $X_{i}$ and $X_{i}^{\prime}$ is smooth (see [Hac12, Section 3]). The W-surface $X_{2}^{\prime}$ has a minimal model and requires only flips to obtain the KSBA replacement. Then this also holds for the $\mathrm{W}$-surface $X_{2}$ since its $\mathbb{Q}$-Gorenstein deformation space is smooth and contains that of $X_{2}^{\prime}$.

All in all, to verify that Conjecture 3.4 is true, we only need to verify it for W-surfaces $X_{1}$ which contain an extremal P-resolution over a WW singularity, so that it contains no other Wahl singularities out of this extremal P-resolution. That is the importance of Theorems 1.2 and 1.3, which will be proved in the next two sections.

\section{Proof of Theorem 1.2}

In this section, we essentially prove that the wormhole conjecture is valid for nonrational surfaces with nef canonical class and with an extremal P-resolution whose middle curve becomes 


\section{G. URzÚa AND N. VilcheS}

a $(-1)$-curve in the minimal resolution. So the only possible counterexamples might come from extremal P-resolutions where the proper transform of the exceptional curve becomes a $(-m)$ curve with $m \geqslant 2$. At first, these seem to be too special over a wormhole singularity, but they turn out to be chaotic. In the next section, we manage to prove the conjecture only for $m=2$ in a special situation.

Throughout this section, we assume the hypotheses of Theorem 1.2, which we now recall. Let $Y$ be a normal projective surface with one cyclic quotient singularity $(Q \in Y)$ which is smooth everywhere else. We assume that the minimal resolution of $Y$ is not ruled and that $Q$ is a wormhole singularity; that is, it admits two extremal P-resolutions $f_{i}^{+}:\left(C_{i} \subseteq X_{i}\right) \rightarrow(Q \in Y)$, for $i=1,2$. In addition we assume the following:

- The strict transform in the minimal resolution of $X_{2}$ of the exceptional curve $C_{2}$ for the extremal P-resolution in $X_{2}$ is a $\mathbb{P}^{1}$ with self-intersection -1 .

- The canonical class $K_{X_{1}}$ is nef.

- Both surfaces $X_{i}$ admit $\mathbb{Q}$-Gorenstein smoothings $\left(X_{i} \subseteq \mathcal{X}_{i}\right) \rightarrow(0 \in \mathbb{D})$; that is, they are W-surfaces.

We want to prove that $K_{X_{2}}$ is nef. This implies that the family $\left(X_{i} \subseteq \mathcal{X}_{i}\right) \rightarrow(0 \in \mathbb{D})$ has nef canonical class (see, for example, [Urz16b, Sect. 2]).

Let $\frac{1}{\Delta}(1, \Omega)=(Q \in Y)$ and $\Delta / \Omega=\left[f_{s}, \ldots, f_{1}\right]-1-\left[e_{1}, \ldots, e_{r}\right]$ be the numerical data of the extremal P-resolution $X_{2} \rightarrow Y$. Let $\sigma: \tilde{X}_{2} \rightarrow X_{2}$ be the minimal resolution of $X_{2}$ over the singularities $P_{1}$ and $P_{2}$. Let $E_{i}$ and $F_{j}$, respectively, be the copies of $\mathbb{P}^{1}$ which resolve them. In this way, we have $E_{i}^{2}=-e_{i}$ and $F_{j}^{2}=-f_{j}$.

Let us assume that $K_{X_{2}}$ is not nef. By hypothesis, we have the existence of $\left(X_{2} \subseteq \mathcal{X}_{2}\right) \rightarrow$ $\left(0 \in \mathbb{D}\right.$ ), and so we know that there is a curve $\Gamma \simeq \mathbb{P}^{1}$ in $X_{2}$ such that $K_{X_{2}} \cdot \Gamma<0$ (see, for example, [Urz16b, Section 2]). Since $X_{2}$ is not ruled, we can assume $\Gamma^{2}<0$, and $\left(\Gamma \subset X_{2} \subseteq\right.$ $\left.\mathcal{X}_{2}\right) \rightarrow(Q \in Y \subset \mathcal{Y})$ is an extremal neighborhood of type mk1A or mk2A. In this way, the curve $\Gamma$ has a very special position in relation to the singularities of $X_{2}$. The assumption that $K_{X_{1}}$ is nef also gives more constraints, which can be summarized as follows:

- Necessarily, $\Gamma$ intersects $\left(f_{1}^{+}\right)^{-1}(Q)$ since otherwise $\Gamma$ would be negative for $K_{X_{1}}$.

- The curve $\Gamma$ cannot intersect $C_{2}$ outside the singularities $P_{1}$ and $P_{2}$ since otherwise we can contract $\Gamma$ in $\tilde{X}_{2}$, producing a surface $X^{\prime}$ and a curve $C_{2}$ with $K_{X^{\prime}} \cdot C_{2}<-1$. But this is contrary to our assumption that $\tilde{X}_{2}$ is not ruled (and so it has a minimal model).

- As we have an mk1A or $m k 2 A$ situation, the curve $\Gamma$ in $\tilde{X}_{2}$ can touch one Wahl chain transversally at one point, or both chains transversally at the ends of each. The first option is not possible since either $\Gamma$ becomes a negative curve for $K_{X_{1}}$ or we have contradiction with the not ruled assumption.

Therefore, the curve $\Gamma$ can only intersect $F_{1}, F_{s}$ and $E_{1}, E_{r}$ in an mk2A situation (four possibilities). In the next arguments, we will strongly use the discrepancies of the two Wahl singularities. We recall that

$$
K_{X_{2}} \cdot \Gamma=\left(K_{\tilde{X}_{2}}-\sum_{a} k_{a} E_{a}-\sum_{b} l_{b} F_{b}\right) \cdot \Gamma=-1-k_{i}-l_{j},
$$

where $k_{a}$ and $l_{b}$ are the discrepancies of the corresponding divisors and $i=1, r$ and $j=1, s$ are the only possibilities. We can easily discard two of the four possibilities: 


\section{ON WORMHOLES IN THE MODULI SPACE OF SURFACES}

- If $\Gamma$ intersects $E_{1}$ and $F_{1}$, then $K_{X_{1}} \cdot \Gamma=K_{X_{1}} \cdot C_{2}>0$ because both curves become $(-1)$-curves in the minimal resolution.

- If $\Gamma$ intersects $E_{r}$ and $F_{s}$, then the extremal P-resolution on $X_{1}$ must have two singularities (since otherwise $\Gamma$ will intersect the singularity only once, and so it will be negative for $K_{X_{1}}$ ). In this way, and as in the proof of Theorem 2.5, it follows that $\Gamma$ must intersect the extreme curves of the two chains from the minimal resolution of $X_{1}$. By the same result, we know that in this case, the strict transform of $C_{1}$ in the minimal resolution of $X_{1}$ is a (-1)-curve. We also know that discrepancies at the end of a Wahl chain add up to -1 . Therefore, we obtain

$$
K_{X_{1}} \cdot \Gamma+K_{X_{1}} \cdot C_{1}=0,
$$

but $K_{X_{1}} \cdot C_{1}>0$, and so we have a contradiction.

The third and fourth possibilities are symmetric, so without loss of generality, we assume that $\Gamma$ is intersecting $E_{1}$ and $F_{s}$ as in Figure 7.

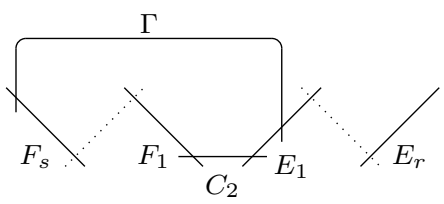

FiguRE 7 . The potential bad curve $\Gamma$ in $\tilde{X}_{2}$

We note that we must have $r>1$ since $r=1$ would give a $\Gamma$ intersecting $E_{r}=E_{1}$ and $F_{s}$, but this case was ruled out above.

Proposition 4.1. Let $Z$ be a normal projective surface and $P_{1}, P_{2} \in Z$ the only singular points, which are Wahl singularities, and let $\sigma: \tilde{Z} \rightarrow Z$ be the minimal resolution of $Z$, which is not ruled. Assume that there exist (-1)-curves $C$ and $\Gamma$ such that on the minimal resolution, we have the configuration given by Figure 7 (taking $C=C_{2}$ ), where $E_{1}, \ldots, E_{r}$ and $F_{1}, \ldots, F_{s}$ are the resolutions of $P_{1}$ and $P_{2}$. Also assume $r>1$. Then, we cannot have simultaneously $K_{Z} \cdot C>0$ and $K_{Z} \cdot \Gamma<0$.

This proposition allows us to finish the proof of Theorem 1.2. It will also be used in the next section.

Proof of Theorem 1.2. Assume that $K_{X_{2}}$ is not nef. As we discussed above, we get a rational curve $\Gamma$, which is negative on $K_{X_{2}}$ and positive on $K_{X_{1}}$ and which gives us Figure 7 on the minimal resolution (with $r>1$ ). We then have $K_{X_{2}} \cdot C_{2}>0$ and $K_{X_{2}} \cdot \Gamma<0$, which contradicts Proposition 4.1 with $Z=X_{2}$ and $C=C_{2}$.

The proof of Proposition 4.1 will be achieved by means of the next few lemmas.

LEMMA 4.2. We must have $s>1$.

Proof. If $s=1$, then $K_{Z} \cdot \Gamma=K_{Z} \cdot C>0$.

Lemma 4.3. We must have $e_{r}=f_{s}=2$.

Proof. As $r, s>1$, exactly one of the values $e_{1}$ and $e_{r}$ is 2 , and the same holds for $f_{1}$ and $f_{s}$. We will verify that the other 3 cases for $e_{r}$ and $f_{s}$ are impossible: 


\section{G. URzÚa AND N. VilcheS}

- If $e_{1}=f_{1}=2$, then contracting $E_{1}, C, F_{1}$ in the configuration, we obtain a $\mathbb{P}^{1}$ with self-intersection equal to 0 . But this gives a contradiction with the not ruled assumption on $\tilde{Z}$.

- If $e_{1}=f_{s}=2$, then the argument against is analogous to the previous one with $E_{1}, \Gamma, F_{s}$.

- Let $e_{r}=f_{1}=2$. We have $K_{Z} \cdot \Gamma=-1-k_{1}-l_{s}$. When we compute the values of $\delta_{i}$ for $\left[e_{1}, \ldots, e_{r}\right]$, we obtain that $\delta_{1}<\delta_{r}$, and so

$$
k_{1}=-1+\frac{\delta_{1}}{\delta_{1}+\delta_{r}}<-1+\frac{\delta_{1}}{\delta_{1}+\delta_{1}}=-\frac{1}{2} .
$$

An analogous argument shows that $l_{s}<-\frac{1}{2}$, and so

$$
K_{Z} \cdot \Gamma>-1+\frac{1}{2}+\frac{1}{2}=0 .
$$

This shows that the only option is $e_{r}=f_{s}=2$.

The argument above used a very simple observation on the discrepancies of Wahl singularities. To continue the proof of Proposition 4.1, we need a more general statement on these discrepancies.

Lemma 4.4. Let $\left[b_{1}, \ldots, b_{t}\right]$ be a Wahl singularity, assume $t \geqslant 2$ and $b_{t}=2$, and let us denote its discrepancies by $m_{1}, \ldots, m_{t}$. Then we have the following bounds:

(Type $M$ ) If $b_{2}=b_{3}=\cdots=b_{t}$, then $m_{1}=-1+1 /\left(b_{1}-2\right)$ and $m_{t}=-1 /\left(b_{1}-2\right)$.

(Type $B$ ) Otherwise, $m_{1}=-1+\mu$ and $m_{t}=-\mu$, where $1 / b_{1}<\mu<1 /\left(b_{1}-1\right)$.

Proof. We will again use the $\delta_{i}$ as in equation 2.5.

Type $M$. Every such singularity comes from [4] by adding 2 s to the right. In this way, $\delta_{1}=1$, $\delta_{2}=2, \ldots, \delta_{t}=t$. Then the discrepancies are $m_{1}=-1+1 /(t+1)$ and $m_{t}=-1+t /(t+1)$. As $b_{1}=t+3$, we get what we wanted.

Type $B$. Let us say that $b_{1}=p+2$. Eliminating the 2 s on the right, we obtain $\left[2, b_{2}, \ldots, b_{t-p}\right]$ with $b_{t-p}>2$. In this way, $\delta_{1}>\delta_{t-p}$ because the first entry is a 2 . Adding back the $2 \mathrm{~s}$ on the right, we get

$$
\delta_{t-p+i}=\delta_{t-p}+i \delta_{1}, \quad i=0, \ldots, p .
$$

In particular, $\delta_{t}=\delta_{t-p}+p \delta_{1}$. Hence, if $\mu=\delta_{1} /\left(\delta_{1}+\delta_{t}\right)$, then

$$
m_{1}=-1+\frac{\delta_{1}}{\delta_{1}+\delta_{t}}=-1+\mu \quad \text { and } \quad m_{t}=-1+\frac{\delta_{t}}{\delta_{1}+\delta_{t}}=-\frac{\delta_{1}}{\delta_{1}+\delta_{t}}=-\mu .
$$

It is then enough to bound $\mu$. As $\delta_{1}>\delta_{t-p}$, we have

$$
\mu>\frac{\delta_{1}}{\delta_{1}+\left(\delta_{1}+p \delta_{1}\right)}=\frac{1}{p+2}=\frac{1}{b_{1}} .
$$

On the other hand, as all $\delta_{i}$ are positive,

$$
\mu=\frac{1}{p+1} \cdot \frac{(p+1) \delta_{1}}{(p+1) \delta_{1}+\delta_{t-p}}<\frac{1}{p+1}=\frac{1}{b_{1}-1} .
$$

These two bounds give $1 / b_{1}<\mu<1 /\left(b_{1}-1\right)$.

We now continue the proof of Proposition 4.1.

Lemma 4.5. Necessarily, $\left[e_{1}, \ldots, e_{r}\right]$ must be of type $M$. Moreover, if $\left[f_{1}, \ldots, f_{s}\right]$ is of type $B$, then $e_{1}=f_{1}+1$. If $\left[f_{1}, \ldots, f_{s}\right]$ is of type $M$, then $e_{1}=f_{1}-1$. 


\section{ON WORMHOLES IN THE MODULI SPACE OF SURFACES}

Proof. The basic idea is to see what happens to $E_{1}$ in $\tilde{Z}$ after we contract all possible $(-1)$ curves. We can contract $\Gamma$ and $C$ and then all $(-2)$-curves at the end of the $F_{j}$ chain $F_{s}, \ldots, F_{s^{\prime}}$. This will impose conditions on $e_{1}$ and $f_{1}$, which will allow us to bound the discrepancies involved in $K_{Z} \cdot \Gamma$.

We are going to analyze the four possible cases, which depend on the type $B$ or $M$ of the singularities.

Type $(B B)$. If $\left[e_{1}, \ldots, e_{r}\right]$ and $\left[f_{1}, \ldots, f_{s}\right]$ are of type $B$, then we have $f_{1}-2$ entries 2 starting with $f_{s}$, and so the curve $E_{1}$ will have self-intersection $-e_{1}+1+1+\left(f_{1}-2\right)=-e_{1}+f_{1}$ after we contract $\Gamma, C$, and $\left\{F_{s}, \ldots, F_{s^{\prime}}\right\}$. Because of our not ruled assumption on $\tilde{Z}$, we must have $e_{1} \geqslant f_{1}+1$. By Lemma 4.4, we have

$$
k_{1}<-1+\frac{1}{e_{1}-1}, \quad l_{s}<-\frac{1}{f_{1}} .
$$

Therefore,

$$
K_{Z} \cdot \Gamma>-1+1-\frac{1}{e_{1}-1}+\frac{1}{f_{1}}=\frac{e_{1}-1-f_{1}}{\left(e_{1}-1\right) f_{1}} \geqslant 0
$$

since $e_{1} \geqslant f_{1}+1$.

Type $(M B)$. If $\left[f_{1}, \ldots, f_{s}\right]$ is of type $M$ and $\left[e_{1}, \ldots, e_{r}\right]$ is of type $B$, then we have $s-1=f_{1}-4$ entries 2 starting with $f_{s}$, and so the curve $E_{1}$ will have self-intersection $-e_{1}+1+1+\left(f_{1}-4\right)=$ $-e_{1}+f_{1}-2$ after we contract $\Gamma, C$, and $\left\{F_{s}, \ldots, F_{s^{\prime}}\right\}$. Again, because of our not ruled assumption on $\tilde{Z}$, we must have $e_{1} \geqslant f_{1}-1$. The bound for $k_{1}$ is as above, while $l_{s}=-1 /\left(f_{1}-2\right)$. In this way,

$$
K_{Z} \cdot \Gamma>-1+1-\frac{1}{e_{1}-1}+\frac{1}{f_{1}-2}=\frac{e_{1}-f_{1}+1}{\left(e_{1}-1\right)\left(f_{1}-2\right)} \geqslant 0
$$

since $e_{1} \geqslant f_{1}-1$.

Type $(B M)$. If $\left[f_{1}, \ldots, f_{s}\right]$ is of type $B$ and $\left[e_{1}, \ldots, e_{r}\right]$ is of type $M$, then we have $f_{1}-2$ entries 2 starting with $f_{s}$, and so the curve $E_{1}$ will have self-intersection $-e_{1}+f_{1}$ after the contractions as above, and so $e_{1} \geqslant f_{1}+1$. By Lemma 4.4 , we can write

$$
k_{1}=-1+\frac{1}{e_{1}-2}, \quad l_{s}<-\frac{1}{f_{1}},
$$

and so

$$
K_{Z} \cdot \Gamma>-1+1-\frac{1}{e_{1}-2}+\frac{1}{f_{1}}=\frac{e_{1}-2-f_{1}}{\left(e_{1}-2\right) f_{1}} .
$$

If $e_{1} \geqslant f_{1}+2$, then $K_{Z} \cdot \Gamma>0$, and so we necessarily get $e_{1}=f_{1}+1$.

Type $(M M)$. If $\left[e_{1}, \ldots, e_{r}\right]$ and $\left[f_{1}, \ldots, f_{s}\right]$ are of type $M$, we have $f_{1}-4$ entries 2 starting with $f_{s}$, and so the curve $E_{1}$ will have self-intersection $-e_{1}+f_{1}-2$ after the contractions as above, and so $e_{1} \geqslant f_{1}-1$. By Lemma 4.4, we have

$$
k_{1}=-1+\frac{1}{e_{1}-2}, \quad l_{1}=-\frac{1}{f_{1}-2},
$$

and so

$$
K_{Z} \cdot \Gamma=-1+1-\frac{1}{e_{1}-2}+\frac{1}{f_{1}-2}=\frac{e_{1}-f_{1}}{\left(e_{1}-2\right)\left(f_{1}-2\right)} .
$$

If $e_{1} \geqslant f_{1}$, then $K_{Z} \cdot \Gamma>0$, and so we necessarily get $e_{1}=f_{1}-1$.

Lemma 4.6. Necessarily, $\left[e_{1}, \ldots, e_{r}\right]$ is of type $M$ and $\left[f_{1}, \ldots, f_{s}\right]$ is of type $B$. 


\section{G. URzÚa AND N. VilcheS}

Proof. By Lemma 4.5, the only other possibility is that $\left[e_{1}, \ldots, e_{r}\right]$ and $\left[f_{1}, \ldots, f_{s}\right]$ are of type $M$, together with $e_{1}=f_{1}-1$. Let $q:=f_{1} \geqslant 5$; we have

$$
\frac{\Delta}{\Omega}=[\underbrace{2, \ldots, 2}_{q-4}, q]-1-[q-1, \underbrace{2, \ldots, 2}_{q-5}] .
$$

We have $r=q-4$ and $s=q-3$. In $\tilde{Z}$, we have a situation as in Figure 8 , where $C^{2}=\Gamma^{2}=-1$, $F_{1}^{2}=-q, E_{1}^{2}=-(q-1)^{2}$, and all the rest are $(-2)$-curves.

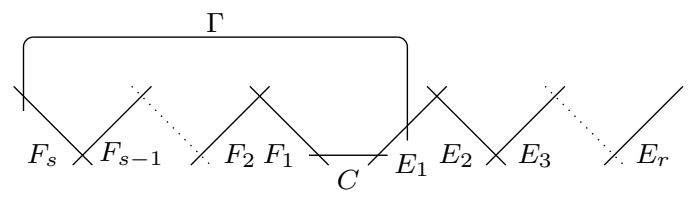

Figure 8. Situation in $\tilde{Z}$, case $M M$

After we contract $\Gamma, C, F_{q-3}, \ldots, F_{2}$, we obtain that $F_{1}$ and $E_{1}$ form a cycle followed by the chain $E_{2}, \ldots, E_{r}$, where $E_{1}^{2}=-1, F_{1}^{2}=-(q-2)$, and all the rest are $(-2)$-curves. We can now contract $E_{1}$, so $F_{1}$ becomes a nodal rational curve and $F_{1}^{2}=-(q-6)$. We now keep contracting $E_{2}, \ldots, E_{r}$, obtaining that

$$
K \cdot F_{1}=(q-6)-2 \cdot(r-1)=(q-6)-2(q-5)=4-q<0
$$

since $q \geqslant 5$. This gives a contradiction since $\tilde{Z}$ is not ruled.

By Lemma 4.6, the only option is $\left[e_{1}, \ldots, e_{r}\right]$ of type $M$ and $\left[f_{1}, \ldots, f_{s}\right]$ of type $B$. Hence we can write

$$
\left[f_{1}, \ldots, f_{s}\right]=[p, f_{2}, \ldots, f_{t}, \underbrace{2, \ldots, 2}_{p-2}],
$$

where $t \geqslant 2$. Canceling the $2 \mathrm{~s}$ on the right, we get $\left[2, f_{2}, \ldots, f_{t}\right]$, which is a Wahl chain again. As its length is at least 2 , we have that $\left[f_{t}, \ldots, f_{2}, 2\right]$ is of type $M$ or $B$. In the final two lemmas of this section, we will say that $\left[f_{1}, \ldots, f_{s}\right]$ is of type $B M$ or $B B$ respectively.

Lemma 4.7. If $\left[e_{1}, \ldots, e_{r}\right]$ is of type $M$, then $\left[f_{1}, \ldots, f_{s}\right]$ must be of type $B B$.

Proof. We assume that $\left[e_{1}, \ldots, e_{r}\right]$ is of type $M$ and $\left[f_{1}, \ldots, f_{s}\right]$ is of type $B M$. Let $p=f_{1}=$ $e_{1}-1 \geqslant 4$. Then we have

$$
\left[e_{1}, \ldots, e_{r}\right]=[p+1, \underbrace{2, \ldots, 2}_{p-3}] \text { and }\left[f_{1}, \ldots, f_{s}\right]=[p, \underbrace{2, \ldots, 2}_{q-5}, q, \underbrace{2, \ldots, 2}_{p-2}] \text {, }
$$

where $q \geqslant 5$. Let $t=q-3$ be the position of the entry equal to $q$. In this way, $E_{1}^{2}=-(p+1)$, $F_{1}^{2}=-p, F_{t}^{2}=-q, C^{2}=\Gamma^{2}=-1$, and all other curves in this situation are $(-2)$-curves.

We will achieve a contradiction by showing that $K \cdot F_{t}$ is eventually negative with $F_{t}$ singular, which goes against the assumption that $\tilde{Z}$ is not ruled. We first contract $C, \Gamma, F_{s}, \ldots, F_{t+1}$. Then $E_{1}$ becomes a $(-1)$-curve. We then contract $E_{1}, \ldots, E_{r}$, and so $F_{1}$ becomes a $(-1)$-curve. If $q>5$, then $F_{t}$ intersects $F_{1}$ only at one point with $F_{t} \cdot F_{1}=p-2$. By contracting $F_{1}, \ldots, F_{t-1}$, we get a singular curve $F_{t}$ with $K \cdot F_{t}=-(p-3)(q-3)-1<0$. If $q=5$, then $F_{t}$ intersects $F_{1}$ at two points, with $F_{t} \cdot F_{1}=1+(p-2)$. After contracting $F_{1}$, we get a singular $F_{t}$ with $K \cdot F_{t}=5-2 p<0$. 


\section{ON WORMHOLES IN THE MODULI SPACE OF SURFACES}

Lemma 4.8. The case $\left[e_{1}, \ldots, e_{r}\right]$ of type $M$ and $\left[f_{1}, \ldots, f_{s}\right]$ of type $B B$ is impossible.

Proof. Let $p=f_{1}=e_{1}-1 \geqslant 4$. We can write

$$
\left[e_{1}, \ldots, e_{r}\right]=[p+1, \underbrace{2, \ldots, 2}_{p-3}] \text { and }\left[f_{1}, \ldots, f_{s}\right]=[p, \underbrace{2, \ldots, 2}_{q-3}, \ldots, q, \underbrace{2, \ldots, 2}_{p-2}],
$$

where $q \geqslant 3$. Let $t=s-(p-2)$ be the position of the entry $q$. The contractions that will come are exactly the contractions we perform in Lemma 4.8, but at the end we are contracting $F_{1}, \ldots, F_{q-2}$. The relevant intersection now is

$$
K \cdot F_{t}=(q-p-1)-(q-2)(p-2)=-(p-3)(q-1)-2<0 .
$$

With Lemma 4.8, we finish the proof of Proposition 4.1, and so that of Theorem 1.2.

\section{Proof of Theorem 1.3}

Throughout this section, we assume the hypotheses of Theorem 1.3, which we now recall. Let $Y$ be a normal projective surface with one cyclic quotient singularity $(Q \in Y)$ which is smooth everywhere else. We assume that the minimal resolution of $Y$ is not ruled and that $Q$ is a wormhole singularity; that is, it admits two extremal P-resolutions $f_{i}^{+}:\left(C_{i} \subseteq X_{i}\right) \rightarrow(Q \in Y)$, for $i=1,2$. In addition we assume the following:

- The strict transform in the minimal resolution of $X_{2}$ of the exceptional curve $C_{2}$ for the extremal P-resolution in $X_{2}$ is a $\mathbb{P}^{1}$ with self-intersection -2 , and $X_{2}$ has only one singularity.

- The canonical class $K_{X_{1}}$ is nef.

- Both surfaces $X_{i}$ admit $\mathbb{Q}$-Gorenstein smoothings $\left(X_{i} \subseteq \mathcal{X}_{i}\right) \rightarrow(0 \in \mathbb{D})$; that is, they are $\mathrm{W}$-surfaces.

We want to prove that we only need flips to run the MMP on $\left(X_{2} \subset \mathcal{X}_{2}\right) \rightarrow(0 \in \mathbb{D})$. Here we cannot guarantee that $K_{X_{2}}$ is nef, we indeed may need some flips, as shown by Example 3.5. The proof will be substantially different from the proof of Theorem 1.2, and lemmas will be given in a more general situation than the one we started with.

LEMMA 5.1. Let $\tilde{Z}$ be a smooth projective surface which is not ruled. Suppose that $\tilde{Z}$ has some chain of smooth rational curves $C, E_{1}, \ldots, E_{r}$, with $C^{2}=-2$ and $E_{i}^{2}=-b_{i}$ with $b_{i} \geqslant 2$, and $\left[b_{1}, \ldots, b_{r}\right]=m^{2} /(m a-1)$ is a Wahl chain. Also suppose that we have a $(-1)$-curve $\Gamma$ which transversely intersects only one $E_{j}$ at one point and also intersects $C$. Then, it follows that $\Gamma$ intersects $C$ transversely at one point, $b_{j} \neq 2$, and $j \neq r$.

This lemma will be useful when we have an mk1A neighborhood via $\Gamma$ over an extremal P-resolution with only one Wahl singularity and a (-2)-curve. We can take $\tilde{Z}$ as the minimal resolution of the singularity and $E_{1}, \ldots, E_{r}$ as the exceptional divisors.

Proof. First note that $K \cdot C=0$ by adjunction. If we blow down $\Gamma$, then the intersection $K \cdot C$ decreases in $\Gamma \cdot C$. But the canonical class must be eventually nef, and so the only possibility is $\Gamma \cdot C=1$.

After blowing down $\Gamma$, we can blow down $C$, and so $E_{j}^{2} \leqslant-3$ as $\tilde{Z}$ is not ruled; that is, $b_{j} \neq 2$.

Now suppose $j=r$. Since $b_{j}>2$, we have $b_{1}=2$ (or $r=1$, where $b_{1}=b_{j}=4$, which leads to a straightforward contradiction). We have two possibilities: 


\section{G. URzÚa AND N. VilcheS}

- If $\left[b_{r}, \ldots, b_{1}\right]$ is a Wahl singularity of type $M$, so that $\left[b_{1}, \ldots, b_{r}\right]=[2, \ldots, 2, r+3]$, we can blow down $\Gamma, C$, and $E_{1}, E_{2}, \ldots, E_{r-1}$. We get a nodal curve $E_{r}$ with $K \cdot E_{r}=-1$, which gives a contradiction since $\tilde{Z}$ is not ruled.

- If $\left[b_{r}, \ldots, b_{1}\right]$ is a Wahl singularity of type $B$, so that

$$
\left[b_{1}, \ldots, b_{r}\right]=\left[2, \ldots, 2, b_{s+1}, \ldots, b_{r-1}, s+2\right],
$$

we can blow down $\Gamma, C$, and $E_{1}, \ldots, E_{s}$. Thus, we get $K \cdot E_{r}=-2$, which again gives a contradiction.

It follows that $j \neq r$.

The next lemma will be useful to control mk1A neighborhoods which will appear in the proof of Theorem 1.3. Roughly speaking, when we run the MMP on $\left(X_{2} \subset \mathcal{X}_{2}\right) \rightarrow(0 \in \mathbb{D})$, we can get an extremal P-resolution with either two singularities and a $(-1)$-curve in the middle, or just one singularity with a (-2)-curve.

Lemma 5.2. Assume that the hypotheses of Lemma 5.1 hold. Let $Z$ be the surface obtained by contracting $E_{1}, \ldots, E_{r}$, and assume that $Z$ admits a $\mathbb{Q}$-Gorenstein smoothing $(Z \subset \mathcal{Z}) \rightarrow(0 \in \mathbb{D})$. Then $\Gamma \subseteq Z$ induces an mk1A neighborhood which must be of flipping type. The resulting extremal P-resolution after the flip must have either two Wahl singularities with a (-1)-curve in the middle or one Wahl singularity with a (-2)-curve.

Proof. Let $\sigma: \tilde{Z} \rightarrow Z$ be the minimal resolution of $Z$. The curve $\Gamma$ induces an mk1A neighborhood on $Z$. Note that Lemma 5.1 says that we must have $b_{j}>2$. Hence we have the mk1A neighborhood $(\Gamma \subset Z) \rightarrow(Q \in Y)=\frac{1}{\Delta}(1, \Omega)$, where every entry of

$$
\frac{\Delta}{\Omega}=\left[b_{1}, \ldots, b_{j}-1, \ldots, b_{r}\right]
$$

is at least 2 . Since $\left[b_{1}, \ldots, b_{r}\right]$ is a Wahl singularity, $\sum b_{i}=3 r+1$, and then the sum of the entries of $\Delta / \Omega$ is $3 r$. This proves that $\frac{1}{\Delta}(1, \Omega)$ is not a Wahl singularity, and then we have a flipping mk1A (see Subsection 3.1).

After we flip, we obtain a new $\mathrm{W}$-surface $Z^{\prime}$, together with a extremal P-resolution over $(Q \in Y)$. Hence can apply Theorem 2.5 to it since we computed before the sum of the entries of the minimal resolution of $(Q \in Y)$. We get that if the new extremal P-resolution has one singularity, then the self-intersection of the flipping curve (on the new minimal resolution) must be -2 ; if there are two singularities, then this self-intersection must be -1 (on the new minimal resolution).

The proof of Theorem 1.3 will be based on a repeated use of Lemma 5.2. We will need to control the new outcomes from Lemma 5.2. For that, we give a definition for the two cases.

Definition 5.3. Let $Y$ be a normal projective surface with one cyclic quotient singularity $Q \in Y$. We name the following extremal P-resolutions $(C \subset Z) \rightarrow(Q \in Y)$ as follows:

(Type $(-1))$ The surface $Z$ has two singularities, and the strict transform of $C$ in the minimal resolution of $Z$ is a $(-1)$-curve.

(Type $(-2)$ ) The surface $Z$ has one singularity, and the strict transform of $C$ in the minimal resolution of $Z$ is a $(-2)$-curve.

We are not assuming that $K_{Z}$ is ample, we only require $C \cdot K_{Z}>0$. 


\section{ON WORMHOLES IN THE MODULI SPACE OF SURFACES}

Lemma 5.4. Let us consider the hypotheses of Theorem 1.3. Let $Z_{1}$ be the $W$-surface $X_{2}$. Assume that we have run the MMP on the $W$-surfaces $Z_{1}, \ldots, Z_{m}$ so that the flip from $\left(\Gamma_{i} \subset Z_{i}\right)$ to $\left(C_{i+1} \subset Z_{i+1}\right)$ always comes from a type(-2) extremal P-resolution as in Lemma 5.2. In addition, assume that $K_{Z_{m}}$ is not nef. Then the only possible $m k 1 A$ for $Z_{m}$ is the one described in Lemma 5.1.

Proof. If the conclusion is false, we have a curve $\Gamma_{m} \subset Z_{m}$ that is a (-1)-curve in the minimal resolution $\tilde{Z}_{m}$, is disjoint from $C_{m}$, and intersects only $E_{i}$ transversally at one point. We note that the flipping curves $\Gamma_{i} \subset Z_{i}$ satisfy Lemma 5.1. As none of the $Z_{i}$, and in particular $Z_{m}$, are ruled, the curves $\Gamma_{m-1}$ and $\Gamma_{m}$ are disjoint in $\tilde{Z}_{m}$. Hence $\Gamma_{m}$ is again a $(-1)$-curve in $\tilde{Z}_{m-1}$. Inductively, we obtain a (-1)-curve $\Gamma_{m}$ in $\tilde{Z}_{1}$ which is disjoint from $C_{1}$ and only intersects some $E_{j}$ transversally at one point. We now go to $X_{1}$. Since $X_{1}$ is not ruled, the curve $\Gamma_{m}$ must be a $(-1)$-curve in $\tilde{X}_{1}$ intersecting only one exceptional curve of $\tilde{X}_{1} \rightarrow X_{1}$ transversally at one point. But that is not possible since then $K_{X_{1}} \cdot \Gamma_{m}<0$. Therefore the curve $\Gamma_{m}$ must intersect $C_{m}$, and so we are in the situation of Lemma 5.1.

Lemma 5.5. Let us consider the hypotheses of Theorem 1.3. Let $Z_{1}$ be the $W$-surface $X_{2}$. Assume that we have run the $M M P$ on $W$-surfaces $Z_{1}, \ldots, Z_{m}$ so that the flip from $\left(\Gamma_{i} \subset Z_{i}\right)$ to $\left(C_{i+1} \subset Z_{i+1}\right)$ always comes from a type(-2) extremal P-resolution as in Lemma 5.2 for $i=1, \ldots, m-2$, and the last one is a type(-1). In addition, assume that $K_{Z_{m}}$ is not nef. Then it is not possible to have an $\mathrm{mk} 1 \mathrm{~A}$ neighborhood for $Z_{m}$.

Proof. The proof is similar to that of Lemma 5.4. A potential $\Gamma_{m} \subset Z_{m}$ defining an mk1A will not intersect $C_{m}, C_{m-1}, \ldots, C_{1}$. Hence it will survive untouched until reaching $Z_{1}$, giving an mk1A neighborhood of $Z_{1}$, and in particular it will be a negative curve, but we know that this is not possible since $K_{Z_{1}}$ is nef. It is key that the surfaces involved are not ruled, so that $(-1)$-curves remain disjoint.

We now show a key step to rule out certain mk2A neighborhoods. After that, we will have everything needed to give a proof for Theorem 1.3.

LEMmA 5.6. Let $Z$ be a normal projective surface and $Q_{1}$ and $Q_{2}$ the only singular points on $Z$. Assume that there is a (-1)-curve $D$ passing through $Q_{1}$ and $Q_{2}$ such that $(D \subset Z) \rightarrow(Q \in Y)$ is an extremal P-resolution.

Let $\sigma: \tilde{Z} \rightarrow Z$ be the minimal resolution of $Z$, which is not ruled, with $F_{1}, \ldots, F_{s}$ and $G_{1}, \ldots, G_{t}$ the exceptional divisors for $Q_{1}$ and $Q_{2}$. Suppose that we have two $(-1)$-curves $B$ and $\Gamma$ on $Z$ such that on $\tilde{Z}$, the configuration is as in Figure 9, and $B$ intersects transversally other curves than $G_{t}$ and $F_{s}$ in the set $\left\{F_{i}, G_{j}\right\}$ in a point. Then $\Gamma \cdot K_{Z} \geqslant 0$.

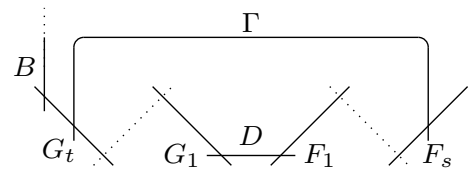

Figure 9. Configuration on $\tilde{Z}$

Proof. Let us assume that we have such a configuration of curves. Let $f_{1}, \ldots, f_{s}, g_{1}, \ldots, g_{t}$ be such that $F_{i}^{2}=-f_{i}$ and $G_{j}^{2}=-g_{j}$. We have $g_{t} \geqslant 3$ because, otherwise, after contracting $B$ and $\Gamma$, the curve $G_{t}$ is a $\mathbb{P}^{1}$ with $G_{t}^{2}=0$, but $\tilde{Z}$ is not ruled. If $f_{s}>2$, then $K_{Z} \cdot \Gamma>0$ because of the discrepancies. So, let us assume $f_{s}=2$. In particular, $s \geqslant 2$. 


\section{G. URzÚa AND N. VilcheS}

Say that $t=1$, and so $g_{t}=4$. Then if we contract $B, D, \Gamma$, we have $G_{t}^{2}=-1$. But we also have $F_{s}^{2}=-1$, and they intersect, which gives a contradiction with the $\tilde{Z}$ not ruled hypothesis. Therefore, we have $t \geqslant 2$, and with that $g_{1}=2$.

Note that in all contractions below, we can never have a chain $[1,2, \ldots, 2,1]$ by the not ruled hypothesis.

Let $p:=f_{1}$ and $q:=g_{t}$, and let $\left\{k_{1}, \ldots, k_{s}\right\}$ and $\left\{l_{1}, \ldots, l_{t}\right\}$ be the corresponding discrepancies. We have the following four cases:

- If $\left[f_{1}, \ldots, f_{s}\right]$ and $\left[g_{t}, \ldots, g_{1}\right]$ are of type $B$, then there are $p-2(-2)$-curves starting with $F_{s}$. Because of the change of self-intersection of $G_{t}$ after contracting $B, \Gamma$, and the $p-2(-2)$ curves, we have $q \geqslant p+1$. By Lemma 4.4, we have

$$
K \cdot \Gamma=-1-k_{s}-l_{t}>-1+\frac{1}{p}+1-\frac{1}{q-1}=\frac{q-p-1}{p(q-1)} \geqslant 0 .
$$

- If $\left[f_{1}, \ldots, f_{s}\right]$ is of type $B$ and $\left[g_{t}, \ldots, g_{1}\right]$ is of type $M$, we have $p-2(-2)$-curves starting with $F_{s}$, and so $q \geqslant p+1$ just as before. But in addition, we can blow down $D, G_{1}, \ldots, G_{t-1}$, which gives the better restriction $q \geqslant p+2$. In this case, Lemma 4.4 gives

$$
K \cdot \Gamma=-1-k_{s}-l_{t}>-1+\frac{1}{p}+1-\frac{1}{q-2}=\frac{q-p-2}{p(q-2)} \geqslant 0 .
$$

- If $\left[f_{1}, \ldots, f_{s}\right]$ is of type $M$ and $\left[g_{t}, \ldots, g_{1}\right]$ is of type $B$, then we have $p-4(-2)$-curves starting with $F_{s}$, and so $q \geqslant p-1$. This implies

$$
K \cdot \Gamma=-1-k_{s}-l_{t}>-1+\frac{1}{p-2}+1-\frac{1}{q-1}=\frac{q+1-p}{(p-2)(q-1)} \geqslant 0 .
$$

- If both $\left[f_{1}, \ldots, f_{s}\right]$ and $\left[g_{t}, \ldots, g_{1}\right]$ are of type $M$, we obtain $q \geqslant p-1$ just as done before. Contracting $D$ and $G_{1}, \ldots, G_{t-1}$, we get $q>p-1$. We also have

$$
K \cdot D=-1+k_{1}+l_{1}=-\frac{1}{p-2}+\frac{1}{q-2}=\frac{p-q}{(p-2)(q-2)}>0,
$$

and so $p>q$. But then $p>q>p-1$, which is not possible.

We now finish the proof of Theorem 1.3.

Let $Z_{1}:=X_{2}$. If $K_{Z_{1}}$ is nef, then we are done. If not, then by Lemma 5.4, we must have an mk1A neighborhood as in Lemma 5.1. Using Lemma 5.2, we can now apply the flip and get $Z_{2}$, which sits in two possible situations: type(-1) or type(-2).

We now assume that we have a chain of flips giving only type(-2), or a chain giving type(-2) followed by one type(-1).

If we only have type(-2), then by Lemma 5.4 , either $K$ is nef, or the new mk1A neighborhood can only be as in Lemma 5.1, and we continue.

If we only have type(-2) and one last type(-1), then we cannot have an mk1A neighborhood by Lemma 5.5. And so either $K$ is nef, or we have a mk2A neighborhood. Then by Lemma 5.6, we can only have a $\Gamma$ intersecting $G_{1}$ and $F_{1}$ (this is not possible since $K \cdot D>0$ ), or $F_{1}$ and $G_{t}$, or $G_{1}$ and $F_{s}$. Note that in the last two cases, we have $t>1$ or $s>1$ since otherwise we can use Lemma 5.6 or have $K \cdot D>0$. Therefore we can apply Proposition 4.1 to deduce that an mk2A neighborhood is impossible, and so $K$ must be nef. This process must end in finitely many steps, so we are done. 


\section{ON WORMHOLES IN THE MODULI SPACE OF SURFACES}

\section{Open questions}

6.1. Topological type of surfaces in a wormhole. Let us start with a couple of examples. Consider a general rational elliptic surface $Z \rightarrow \mathbb{P}^{1}$ with sections and no (-1)-curves in the fibers. Hence any section is a $(-1)$-curve. Let $F_{E}$ and $F_{G}$ be two nodal $I_{1}$-fibers, and let $S$ be a section of $Z \rightarrow \mathbb{P}^{1}$. We blow up $s$ times over the node in $F_{E}$ and $r$ times over the node in $F_{G}$ to obtain a surface $\tilde{X}_{1}$ with Wahl chains $\left[F_{E}, E_{1}, \ldots, E_{s-1}\right]=[3+s, 2, \ldots, 2]$ and $\left[F_{G}, G_{1}, \ldots, G_{r-1}\right]=$ $[3+r, 2, \ldots, 2]$. The contraction of both of these produces a $\mathrm{W}$-surface $X_{1}$ (see [Urz16a, Theorem $4.2]$ ), and the general fibers are either Enriques surfaces (if $r=s=1$ ) or elliptic surfaces of Kodaira dimension 1. In fact, one can prove that the general fiber is an elliptic fibration over $\mathbb{P}^{1}$ with $p_{g}=q=0$ and that there are two multiple fibers of multiplicities $s$ and $r$, and so the fundamental group of $X_{1}$ is $\mathbb{Z} / \operatorname{gcd}(r, s)$. Hence although these are not degenerations of surfaces of general type, they will be useful to see that wormholes may change the topology of the general fibers.

The curve $S$ defines an extremal P-resolution on $X_{1}$. Let us consider the chain of curves $E_{s-1}, \ldots, E_{1}, F_{E}, S, F_{G}, G_{1}, \ldots, G_{r-1}$. Their contraction defines the cyclic quotient singularity $(Q \in Y)$ given by

$$
[2, \ldots, 2,2+s, 2+r, 2, \ldots, 2],
$$

whose dual continued fraction is $[s+1, \overline{2}, \ldots, 2,3,2, \ldots, \overline{2}, r+1]$, where the numbers of $2 \mathrm{~s}$ are $s-1$ (on the left) and $r-1$ (on the right), and we mark with bars the position of the pair which produces the extremal P-resolution indicated above. (The cases $s=1$ or $r=1$ are a bit different, as the reader may check.) We want to check whether $(Q \in Y)$ is a wormhole singularity, and so we are looking for another pair. A quick verification shows that $r>3$ or $s>3$ do not work. For the few cases left, and up to reordering, the only wormhole singularities are $[4, \overline{2}, \underline{2}, 3, \overline{2}, \underline{3}]$ and $[\underline{4}, \overline{2}, \underline{2}, \overline{3}, 2]$, which correspond to the initial extremal P-resolutions (I) $[2,2,6]-1-[5,2]$ and (II) $[2,2,6]-1-[4]$, respectively.

Case (I). In this case, the new extremal P-resolution is $[2,2,5,4]-2$. Let $X_{2}$ be the corresponding W-surface. The curve $G_{2}$ is now a flipping curve, and after the flip, we obtain a Wsurface $X_{2}^{\prime}$ with extremal P-resolution $[2,2,6]-1-[4]$. Therefore, the canonical class now is nef. The general fiber of $X_{1}$ gives an elliptic surface with fundamental group of order $\operatorname{gcd}(4,3)=1$, but the general fiber of $X_{2}^{\prime}$ has fundamental group of order $\operatorname{gcd}(4,2)=2$. Thus they are not homeomorphic.

Case (II). In this case, the new extremal P-resolution is $2-[2,5,3]$. Let $X_{2}$ be the corresponding $\mathrm{W}$-surface. The curve $E_{3}$ is now a flipping curve, and after the flip, we obtain a W-surface $X_{2}^{\prime}$ with extremal P-resolution [2,5]-1- [4], and so the canonical class is now nef. The general fiber of $X_{1}$ gives an elliptic surface with fundamental group of order $\operatorname{gcd}(4,2)=2$, but the general fiber of $X_{2}^{\prime}$ is simply connected since $\operatorname{gcd}(3,2)=1$. Thus they are not homeomorphic as well.

However, in many cases, wormholes produce surfaces with isomorphic fundamental groups. Let us consider a wormhole situation from $X_{1}$ to $X_{2}$ where both have two Wahl singularities corresponding to the extremal P-resolutions. Let $d_{i}$ be the greatest common divisor of the indices of the Wahl singularities in $X_{i}$. (If there is one or zero singularity, then $d_{i}=1$.)

Proposition 6.1. If $d_{1}=d_{2}$, then the fundamental groups of the general fibers of $X_{1}$ and $X_{2}$ are isomorphic.

Proof. Let $f_{i}^{+}:\left(C_{i} \subset X_{i}\right) \rightarrow(Q \in Y)$ be the contractions to a wormhole singularity. We are going to use the Seifert-Van Kampen theorem to compare the fundamental groups of the general 


\section{G. URzÚa AND N. VilcheS}

fibers $X_{1, t}$ and $X_{2, t}$. Let $L$ be the link of $(Q \in Y)$. Let $M_{i}$ be the Milnor fiber of the smoothing of $(Q \in Y)$ corresponding to $X_{i}$ (that is, the blowing-down deformation of the $\mathbb{Q}$-Gorenstein smoothing corresponding to the extremal P-resolution in $\left.X_{i}\right)$. Then $\pi_{1}\left(M_{i}\right) \simeq \mathbb{Z} / d_{i}$ and $\pi_{1}(L) \simeq$ $\mathbb{Z} / \Delta$, where $\frac{1}{\Delta}(1, \Omega)=(Q \in Y)$. Let $X_{i}^{0}$ be the complement of $C_{i}$. Then $X_{1}^{0}=X_{2}^{0}=: X$, and we have

$$
\pi_{1}\left(X_{i, t}\right) \simeq\left(\pi_{1}(X) \star \pi_{1}\left(M_{i}\right)\right) / N\left(\alpha \beta^{-1}\right),
$$

where $\alpha$ generates $\pi_{1}(L)$ in $\pi_{1}(X), \beta$ generates $\pi_{1}(L)$ in $\pi_{1}\left(M_{i}\right)$, and $N\left(\alpha \beta^{-1}\right)$ is the smallest normal subgroup containing $\alpha \beta^{-1}$. By [LW86, Lemma 5.1], the morphism induced by the inclusion $\pi_{1}(L) \rightarrow \pi_{1}\left(M_{i}\right)$ is onto. Therefore, if $\pi_{1}(X)=G / R$, where $G$ consists of generators and $R$ of relations, then $\pi_{1}\left(X_{i, t}\right) \simeq G /\left(R, \alpha^{d_{i}}=1\right)$. The claim follows when $d_{1}=d_{2}$.

Corollary 6.2. If $d_{1}=d_{2}=1$, then the general fibers of $X_{1}$ and $X_{2}$ have isomorphic fundamental groups equal to $\pi_{1}\left(\tilde{X}_{1}\right)=\pi_{1}\left(\tilde{X}_{2}\right)=\pi_{1}(Y)$. In particular, if in addition $\tilde{X}_{1}$ is rational, then wormholes produce simply connected surfaces.

Proof. Here, by applying the Seifert-Van Kampen theorem, we have $\pi_{1}\left(X_{i}\right)=\pi_{1}\left(X_{i}^{0}\right) /(\alpha=1)$, but this is what we just computed for $\pi_{1}\left(X_{i, t}\right)$ when $d_{i}=1$ (alternatively, one can use [LP07, Theorem 3]). The other claim holds because we are dealing with rational singularities.

Let us consider rational W-surfaces $X_{i}$ with $d_{1}=d_{2}=1$. Let us assume $K^{2}=1$ so that the general fibers are simply connected Godeaux surfaces. There are plenty of such wormholes in the KSBA compactification of the moduli space of Godeaux surfaces (see, for example, [LP07, Figure 6] for the $[2,2,6]-1-[4])$. By Freedman's classification theorem, the general fibers are homeomorphic as oriented 4-manifolds. On the other hand, Reid conjectures that the moduli space of torsion zero Godeaux surfaces is irreducible, and so all of these wormholed surfaces should be diffeomorphic. Very recently, Dias and Rito proved Reid's conjecture for $\mathbb{Z} / 2$-Godeaux surfaces in [DR20], and so any wormhole in their KSBA compactification with $d_{1}=d_{2}$ (as in Example 3.5) gives diffeomorphic surfaces.

Question 6.3. For a wormhole with $d_{1}=d_{2}$, are the general fibers always diffeomorphic? Are they homeomorphic?

In fact, one can show that $d_{1}=d_{2}$ preserves the homology together with the intersection form, and so if the $X_{i}$ are simply connected, then Freedman's theorem produces a homeomorphism. More on the topology aspects will be part of a sequel to this work. On the other hand, and as we saw above, for the case $d_{1} \neq d_{2}$, we may have nonhomeomorphic surfaces (although the example was not of general type). In [DRU20, Figure 5], we have a wormhole defined by $[2,5]-1-[2,6,2,3]$ in $X_{1}\left(d_{1}=1\right)$ whose $\mathbb{Q}$-Gorenstein smoothing is a $\mathbb{Z} / 2$-Godeaux surface. Its wormholed surface $X_{2}$ has extremal P-resolution $[2,3,5,3]-1-[4]$, and so $d_{2}=2$. If its $\mathbb{Q}$-Gorenstein smoothing has $\pi_{1} \neq \mathbb{Z} / 2$, then it would be $\mathbb{Z} / 4$ by the classification we have for Godeaux surfaces.

We note that for wormholes of general type and with different fundamental groups, we would be crossing distinct components of the moduli space. We expect that there are many.

6.2. What is left to prove the conjecture. In this paper, we introduced the wormhole conjecture, and we proved it for many situations under the assumption that the singular surfaces involved were not rational. Hence we divide the final discussion in two parts. 


\section{ON WORMHOLES IN THE MODULI SPACE OF SURFACES}

Nonrational. Let $X_{1}$ and $X_{2}$ be the W-surfaces in a wormhole, both with an extremal Presolution over a fixed wormhole singularity, and nonsingular out of them. In the list below, we write Wahl- $m$-Wahl for an extremal P-resolution with two Wahl singularities (distinct or equal) and a middle curve whose self-intersection in the minimal resolution is $-m$. If Wahl is dropped, then the point is nonsingular. Using Theorem 2.5 and because we already have Theorems 1.2 and 1.3, the list of pairs of extremal P-resolutions where we do not know the validity of the wormhole conjecture is

(a) Wahl- $m$-Wahl and Wahl- $m$-Wahl for $m \geqslant 2$,

(b) $m$-Wahl and Wahl- $(m-1)-$ Wahl for $m \geqslant 3$,

(c) Wahl- $m$ and $m$-Wahl for $m \geqslant 3$.

For case (c), we will give some combinatorial counterexamples to the wormhole conjecture, although we do not know if they can be realized on a surface.

Example 6.4. Let us assume the existence of a chain $E_{1}, \ldots, E_{9}$ of copies of $\mathbb{P}^{1}$ in a nonsingular surface $Z$ with nef minimal model, where $E_{i}^{2}=-e_{i}$ and

$$
\left[e_{1}, \ldots, e_{9}\right]=[5,2,2,2,8,2,2,2,5] .
$$

Assume that there is a $(-1)$-curve $\Gamma$ intersecting $E_{1}$ twice and transversally, and disjoint from the rest. This $(-1)$-curve does not produce any contradiction with the minimal model of $Z$. The wormhole singularity $[5,2,2,2,10,2,2,2,5]$ admits two obvious extremal P-resolutions: $[5,2,2,2$, $10,2,2,2]-5$ in $X_{1}$ and $5-[2,2,2,10,2,2,2,5]$ in $X_{2}$, so we are in case (c). The curve $\Gamma$ is positive for $K_{X_{1}}$, and not only is it negative for $K_{X_{2}}$, but it induces a divisorial contraction on the deformation of $X_{2}$. In fact,

$$
[n+2, \underbrace{2, \ldots, 2}_{n}, n+5, \underbrace{2, \ldots, 2}_{n}, n+2]
$$

with a (-1)-curve intersecting only the first curve with multiplicity $n-1$ gives infinitely many bad situations. Are any of these counterexamples realizable?

Rational. Here we do not have a feasible strategy to prove the conjecture. But we have many examples verifying it for the invariants $p_{g}=q=0$ and $K^{2}=1,2,3,4$. These examples are constructed as in [LP07] and they have two singularities; they will be part of some future work.

We finish the paper with another open question. Note that a WW singularity (that is, one that admits at least one pair of indices to be a zero continued fraction) has complete freedom in the values of $\delta$. But this freedom is lost for wormhole singularities.

Question 6.5. What are the possible values for $\delta$ in a wormhole singularity?

For $\Delta \leqslant 450$ we have only 64 wormhole singularities $\frac{1}{\Delta}(1, \Omega)$, and the values of $\delta$ are $2,5,10$, $13,17,26,30,37,50$. These values appear with multiplicities $31,18,4,3,3,1,2,1,1$, respectively. If we consider wormholes singularities whose Hirzebruch-Jung continued fraction has at most 18 


\section{G. URzÚA AND N. VilcheS}

entries and whose extremal P-resolutions require no blow-ups, then the values of $\delta$ are

$$
\begin{gathered}
2,5,10,13,17,26,30,34,37,50,53,58,65,68,82,89,101,122,130, \\
145,170,178,185,197,219,222,226,233,257,290,317,325,327,338, \\
350,457,466,520,578,610,738,853,964,986,997,1010,1220,1237, \\
1342,1515,1597,1740,1970,2018,2210,2487,2758,3005,3194,3390, \\
3505,3567,4112,4181,4930,5722,5725,5850,6878,9282 .
\end{gathered}
$$

Moreover,

$$
\begin{gathered}
2,5,10,13,17,26,30,34,37,50,53,58,65,68,82,89,101,122,130, \\
145,178,185,219,222,233,317,327,338,350,457,466,520,578,610, \\
738,853,964,986,997,1010,1220,1237,1342,1515,1597,1740,1970, \\
2018,2210,2487,2758,3005,3194,3390,3505,3567,4112,4181,4930, \\
5722,5725,5850,6305,6878,7298,8020,9282,10670,10946,11482, \\
12190,13669,13848,15049,15650,17602,19710,20917,24418,27030, \\
28657,29822,39338,75025
\end{gathered}
$$

are all the values of $\delta$ for wormholes singularities whose Hirzebruch-Jung continued fraction has at most 25 entries and for which one of the extremal P-resolutions is of type $m$-Wahl with $m=2$.

The following infinite family has the value $\delta=2$ :

$$
\frac{\Delta}{\Omega}=2-[\underbrace{2, \ldots, 2}_{k-2}, 5, k]=[\underbrace{2, \ldots, 2}_{k-1}, k+3]-1-[\underbrace{2, \ldots, 2}_{k-3}, k+1] .
$$

One can compute

$$
[\underbrace{2, \ldots, 2}_{k-2}, 5, k]=\frac{(2 k-1)^{2}}{(2 k-1)(2 k-3)-1},
$$

which gives $\Delta=4 k^{2}, \Omega=(2 k-1)^{2}, \delta=2$. Actually, the case $\delta=2$ can be classified completely through the use of triangulations of polygons, where one changes one diagonal in a "corner quadrilateral" to the other diagonal.

On the other hand, not every natural number appears as the $\delta$ of a wormhole singularity. For instance, $\delta=3$ is not possible. Indeed, say that $\Delta /(\Delta-\Omega)=\left[a_{1}, \ldots, \bar{a}_{\alpha}, \ldots, \bar{a}_{\beta}, \ldots, a_{r}\right]$ has $\delta=3$. We may also assume $a_{0}>1$. Thus, $a_{\alpha}=a_{\beta}=2, \delta / \varepsilon=\left[a_{\alpha+1}, \ldots, a_{\beta-1}\right]$, and $\delta /(\delta-\varepsilon)=\left[a_{\alpha-1}, \ldots, a_{1}, a_{0}, a_{r}, \ldots, a_{\beta+1}\right]$ from case $(\mathrm{A})$. Now, we have $\varepsilon=1$ or 2 , so one of those continued fractions is $3 / 2=[2,2]$. Then the associated triangulation contains $\{\overline{2}, 2,2, \overline{2}\}$, which clearly gives a contradiction.

A better understanding of the wormhole phenomenon on singularities is wanted, to potentially solve the wormhole conjecture and to show topological implications.

\section{ACKNOWLEDGEMENTS}

The results of this article are mainly based on the master's thesis [Vil20]. We would like to thank Jonny Evans for useful discussions and comments, and the referees for constructive and useful suggestions. 


\section{ON WORMHOLES IN THE MODULI SPACE OF SURFACES}

\section{REFERENCES}

CU18 S. Coughlan and G. Urzúa, On Z/Z-Godeaux surfaces, Int. Math. Res. Not. 2018 (2018), no. 18, 5609-5637; doi:10.1093/imrn/rnx049.

DR20 E. Dias and C. Rito, $\mathbb{Z} / 2$-Godeaux surfaces, 2020, arXiv:2009.12645.

DRU20 E. Dias, C. Rito, and G. Urzúa, On degenerations of $\mathbb{Z} / 2$-Godeaux surfaces, 2020, arXiv: 2002.08836 .

EU18 J. D. Evans and G. Urzúa, Antiflips, mutations, and unbounded symplectic embeddings of rational homology balls, Ann. Inst. Fourier (Grenoble), to appear, arXiv:1807.06073.

Hac12 P. Hacking, Compact moduli spaces of surfaces of general type, in Compact Moduli Spaces and Vector Bundles, Contemp. Math., vol. 564 (Amer. Math. Soc., Providence, RI, 2012), 1-18; doi:10.1090/conm/564/11157.

HTU17 P. Hacking, J. Tevelev, and G. Urzúa, Flipping surfaces, J. Algebraic Geom. 26 (2017), no. 2, 279-345; doi:10.1090/jag/682.

KS88 J. Kollár and N.I. Shepherd-Barron, Threefolds and deformations of surface singularities, Invent. Math. 91 (1988), no. 2, 299-338; doi:10.1007/BF01389370.

LN13 Y. Lee and N. Nakayama, Simply connected surfaces of general type in positive characteristic via deformation theory, Proc. Lond. Math. Soc. 106 (2013), no. 2, 225-286; doi:10.1112/plms/ pds033.

LN18__ Grothendieck duality and $\mathbb{Q}$-Gorenstein morphisms, Publ. Res. Inst. Math. Sci. 54 (2018), no. 3, 517-648; doi:10.4171/PRIMS/54-3-3.

LP07 Y. Lee and J. Park, A simply connected surface of general type with $p_{g}=0$ and $K^{2}=2$, Invent. Math. 170 (2007), no. 3, 483-505; doi:10.1007/s00222-007-0069-7.

LW86 E. Looijenga and J. Wahl, Quadratic functions and smoothing surface singularities, Topology 25 (1986), no. 3, 261-291; doi:10.1016/0040-9383(86)90044-3.

Mum61 D. Mumford, The topology of normal singularities of an algebraic surface and a criterion for simplicity, Publ. Math. Inst. Hautes Études Sci. 9 (1961), 5-22; doi:10.1007/BF02698717.

OW77 P. Orlik and P. Wagreich, Algebraic surfaces with $k^{*}$-action, Acta Math. 138 (1977), no. 1-2, 43-81; doi:10.1007/BF02392313.

PPS09a H. Park, J. Park, and D. Shin, A simply connected surface of general type with $p_{g}=0$ and $K^{2}=3$, Geom. Topol. 13 (2009), no. 2, 743-767; doi:10.2140/gt.2009.13.743.

PPS09b_, A simply connected surface of general type with $p_{g}=0$ and $K^{2}=4$, Geom. Topol. 13 (2009), no. 3, 1483-1494; doi:10.2140/gt.2009.13.1483.

PPSU18 H. Park, J. Park, D. Shin, and G. Urzúa, Milnor fibers and symplectic fillings of quotient surface singularities, Adv. Math. 329 (2018), 1156-1230; doi:10.1016/j.aim.2018.03.002.

Rie74 O. Riemenschneider, Deformationen von Quotientensingularitäten (nach zyklischen Gruppen), Math. Ann. 209 (1974), no. 3, 211-248; doi:10.1007/BF01351850.

RTU17 J. Rana, J. Tevelev, and G. Urzúa, The Craighero-Gattazzo surface is simply-connected, Compos. Math. 153 (2017), no. 3, 557-585; doi:10.1112/S0010437X16008125.

Ste91 J. Stevens, On the versal deformation of cyclic quotient singularities, Singularity Theory and its Applications, Part I (Coventry, 1988/1989), Lecture Notes in Math., vol. 1462 (Springer, Berlin, 1991), 302-319; doi:10.1007/BFb0086390.

SU16 A. Stern and G. Urzúa, KSBA surfaces with elliptic quotient singularities, $\pi_{1}=1, p_{g}=0$, and $K^{2}=1,2$, Israel J. Math. 214 (2016), no. 2, 651-673; doi:10.1007/s11856-016-1350-5.

Urz16a G. Urzúa, Identifying neighbors of stable surfaces, Ann. Sc. Norm. Super. Pisa Cl. Sci. (5) 16 (2016), no. 4, 1093-1122.

Urz16b , $\mathbb{Q}$-Gorenstein smoothings of surfaces and degenerations of curves, Rend. Semin. Mat. Univ. Padova 136 (2016), 111-136; doi:10.4171/RSMUP/136-9. 


\section{G. URZÚA AND N. VILChES}

Vil20 N. Vilches, P-resoluciones extremales y agujeros de gusano en espacios de moduli de superficies, 2020, Tesis de Magister, Pontificia Universidad Católica de Chile, available at http://www.mat.uc.cl/archivos/p-resoluciones-extremales-y-agujeros-de-gusanoen-espacios-de-moduli-de-superficies.pdf.

Giancarlo Urzúa urzua@mat.uc.cl

Facultad de Matemáticas, Pontificia Universidad Católica de Chile, Campus San Joaquín, Avenida Vicuña Mackenna 4860, Santiago, Chile

Nicolás Vilches nivilches@math.columbia.edu

Department of Mathematics, Columbia University, 2990 Broadway, New York, NY 10027, USA 